ESAIM: M2AN 46 (2012) 465-489

DOI: $10.1051 / \mathrm{m} 2 \mathrm{an} / 2011148$
ESAIM: Mathematical Modelling and Numerical Analysis

www.esaim-m2an.org

\title{
A MIXED FINITE ELEMENT METHOD FOR DARCY FLOW IN FRACTURED POROUS MEDIA WITH NON-MATCHING GRIDS *
}

\author{
Carlo D'Angelo ${ }^{1}$ And Anna Scotti ${ }^{2}$
}

\begin{abstract}
We consider an incompressible flow problem in a $N$-dimensional fractured porous domain (Darcy's problem). The fracture is represented by a $(N-1)$-dimensional interface, exchanging fluid with the surrounding media. In this paper we consider the lowest-order $\left(\mathbb{R} \mathbb{T}_{0}, \mathbb{P}_{0}\right)$ Raviart-Thomas mixed finite element method for the approximation of the coupled Darcy's flows in the porous media and within the fracture, with independent meshes for the respective domains. This is achieved thanks to an enrichment with discontinuous basis functions on triangles crossed by the fracture and a weak imposition of interface conditions. First, we study the stability and convergence properties of the resulting numerical scheme in the uncoupled case, when the known solution of the fracture problem provides an immersed boundary condition. We detail the implementation issues and discuss the algebraic properties of the associated linear system. Next, we focus on the coupled problem and propose an iterative porous domain/fracture domain iterative method to solve for fluid flow in both the porous media and the fracture and compare the results with those of a traditional monolithic approach. Numerical results are provided confirming convergence rates and algebraic properties predicted by the theory. In particular, we discuss preconditioning and equilibration techniques to make the condition number of the discrete problem independent of the position of the immersed interface. Finally, two and three dimensional simulations of Darcy's flow in different configurations (highly and poorly permeable fracture) are analyzed and discussed.
\end{abstract}

Mathematics Subject Classification. 76S05, 35Q86, 65L60.

Received October 27, 2010. Revised July 6, 2011.

Published online December 19, 2011.

\section{INTRODUCTION}

The numerical approximation of fluid flows in porous media is particularly challenging in presence of strong heterogeneities of the model parameters. This is a known issue of the Darcy's problem in geophysical applications, such as groundwater flows or two-phase flows for oil migration. In fact, the permeability of the considered medium (the ground, or, at a larger scale, a geological basin) may easily span several orders of magnitude. Mixed finite element methods are known to be robust with respect to such heterogeneity, while ensuring mass conservation

Keywords and phrases. Darcy's equation, fractured porous media, mixed finite element, unfitted mesh, fictitious domain, embedded interface, extended finite element.

* Special thanks to Paolo Zunino and Alessio Fumagalli for many useful discussions.

1 MOX-Dip. di Matematica "F. Brioschi" Politecnico di Milano via Bonardi 9, 20133 Milano, Italy. carlo.dangelo@polimi.it

2 MOX-Dip. di Matematica "F. Brioschi" Politecnico di Milano via Bonardi 9, 20133 Milano, Italy. anna.scotti@mail.polimi.it 
properties. However, heterogeneities can be geometrically structured in the form of fractures (thin regions with a different porous structure). In the relevant case of fractures whose thickness is very small compared to the characteristic length of the domain, a reduced model can be set up in which the fracture is represented as an immersed interface. The development and the analysis of such models for the single phase flow have been extensively addressed in $[1,12,15]$, where the fracture flow equations and the proper interface conditions across the fracture have been identified and mixed finite element schemes for the coupled porous medium flow/fracture flow have been proposed.

In the aforementioned works, the computational grid of the porous domain is considered to be matching with the fracture mesh: in practice, the fracture is the (conforming) interface between two mesh blocks. Nonconforming meshes on the interface could be easily dealt with by mortaring [3], however, this does not allow having an immersed interface, where some of the elements of the porous grid may be cut by the fracture. The aim of this work is precisely to extend the already known reduced models of Darcy's flow in fractured media to the case where the porous mesh and the fracture mesh are independent and non-matching. To do this, we adopt the approach of enriching the classical Raviart-Thomas finite element basis on the elements cut by the fracture with discontinuous functions. This XFEM concept is borrowed from the works by Hansbo et al. [5, 6, 14] that focus on the elasticity problem in domains with fractures.

The aim of this study is to provide a flexible tool for handling the fractures and the porous bulk of the considered medium independently. This is of interest in several typical situations. First, it is often difficult to force the computational grid to be conformal with a given fracture network, especially if the latter has a complex geometry. Moreover, there are important applications that require to run multiple simulations with different fracture configurations. Among some relevant instances we mention the geological scenario analysis, more generally the quantification of uncertainty on physical parameters, or the upscaling of random fractured media [7]. In this context, the possibility of running simulations with different fracture geometries while keeping the mesh of the porous domain unchanged is an advantage of the proposed approach.

\section{Formulation OF THE PROBlem}

To make the exposition more clear, we specifically address the $(N=2)$-dimensional case; nevertheless, this assumption will not be a limitation. We consider a bounded open domain $\Omega \subset \mathbb{R}^{2}$ (the porous media or bulk) and a line $\Gamma \subset \Omega$ (the fracture).

For the sake of simplicity, we shall consider the case where $\Gamma$ separates $\Omega$ in two disjoint domains $\Omega_{i}$, $i=1,2$. This is not a strict limitation of the proposed method; however, handling the case of a "partially immersed fracture" is more complex from the analytical point of view (see [2] for a thorough investigation concerning the proper analytical setting of the continuous problem).

We shall denote $\mathbf{n}_{\Gamma}$ a normal unit vector with fixed orientation on $\Gamma$ from $\Omega_{1}$ to $\Omega_{2}$, and $\boldsymbol{\tau}_{\Gamma}$ the tangential unit vector on $\Gamma$ (in $N>2$ dimensions $\boldsymbol{\tau}_{\Gamma}$ will be a $N \times(N-1)$ matrix, whose columns constitute an orthonormal basis for the tangent space at each point $\hat{\mathbf{x}} \in \Gamma)$. We will often refer to the canonical local coordinates $(s, t)$ to map a neighborhood of $\hat{\mathbf{x}} \in \Gamma$ by $\mathbf{x}=\hat{\mathbf{x}}+s \boldsymbol{\tau}_{\Gamma}+t \mathbf{n}_{\Gamma}$ (or, if $N>2, \mathbf{x}=\hat{\mathbf{x}}+\boldsymbol{\tau}_{\Gamma} \boldsymbol{s}+t \mathbf{n}_{\Gamma}$ where $\boldsymbol{s} \in \mathbb{R}^{N-1}$ is the vector of the tangential components).

According to the different porous microstructure of the bulk and the fracture, we consider a permeability tensor field $\mathrm{K}$ in $\Omega$, and assume the permeability tensor in the fracture to be block-diagonal in local coordinates $(s, t)$, i.e.

$$
\mathrm{K}_{\Gamma}=\left[\begin{array}{cc}
\mathrm{K}_{\Gamma, n} & 0 \\
0 & \mathrm{~K}_{\Gamma, \tau}
\end{array}\right],
$$

where $\mathrm{K}_{\Gamma, n}$ is the normal permeability and $\mathrm{K}_{\Gamma, \tau}$ is the tangential permeability tensor.

We assume that the motion of the incompressible fluid in $\Omega$ is governed by the Darcy's equation. In particular, let $\mathbf{u}, p$ be respectively the filtration velocity and the fluid pressure in $\Omega$, and let $\eta=\mathrm{K}^{-1}$. Assume $\partial \Omega=\overline{\Gamma_{D}} \cup \overline{\Gamma_{N}}$, $\Gamma_{D} \cap \Gamma_{N}=\emptyset$, and let the boundary flux $u_{0}: \Gamma_{D} \rightarrow \mathbb{R}$ and the external pressure $p_{0}: \Gamma_{N} \rightarrow \mathbb{R}$ be given. The flow 
equations and boundary conditions read

$$
\begin{array}{llll}
\eta \mathbf{u}+\nabla p=\boldsymbol{f}_{v} & \text { in } \Omega, & \mathbf{u} \cdot \mathbf{n}=u_{0} & \text { on } \Gamma_{D}, \\
\nabla \cdot \mathbf{u}=f_{q} & \text { in } \Omega, & p=p_{0} & \text { on } \Gamma_{N},
\end{array}
$$

where $\boldsymbol{f}_{v}, f_{q}$ are given data (representing for instance gravitational effects and mass sources/sinks inside the domain).

Finally, suitable interface conditions on $\Gamma$ have to be provided. A first example is the following: (i) the fluid pressure in the fracture is the mean value of the fluid pressures on the two sides of the surrounding porous medium, and (ii) the mean normal flow rate is proportional to the pressure jump across $\Gamma$, i.e.

$$
\begin{array}{ll}
0=\{p\}-\hat{p} & \text { on } \Gamma, \\
\eta_{\Gamma}\left\{\mathbf{u} \cdot \mathbf{n}_{\Gamma}\right\}=\llbracket p \rrbracket & \text { on } \Gamma,
\end{array}
$$

where $\hat{p}$ is the fluid pressure in the fracture, $\eta_{\Gamma}=\frac{l_{\Gamma}}{\mathrm{K}_{\Gamma, n}}, l_{\Gamma}$ being the actual fracture thickness, and we adopt the following notations for the jump and the average of any function $u$ that may be discontinuous across $\Gamma$ :

$$
\llbracket u \rrbracket:=u_{1}-u_{2}, \quad\{u\}:=\frac{1}{2}\left(u_{1}+u_{2}\right), \quad \text { where } \quad u_{1,2}(\mathbf{x})=\lim _{\epsilon \rightarrow 0^{ \pm}} u\left(\mathbf{x}-\epsilon \mathbf{n}_{\Gamma}\right) \quad \forall \mathbf{x} \in \Gamma .
$$

This is a particular case $\left(\xi=\frac{1}{2}\right)$ of the following interface conditions [15]

$$
\begin{array}{ll}
\xi \mathbf{u}_{1} \cdot \mathbf{n}_{\Gamma}+(1-\xi) \mathbf{u}_{2} \cdot \mathbf{n}_{\Gamma}=2 \eta_{\Gamma}^{-1}\left(p_{1}-\hat{p}\right) & \text { on } \Gamma, \\
(1-\xi) \mathbf{u}_{1} \cdot \mathbf{n}_{\Gamma}+\xi \mathbf{u}_{2} \cdot \mathbf{n}_{\Gamma}=2 \eta_{\Gamma}^{-1}\left(\hat{p}-p_{2}\right) & \text { on } \Gamma,
\end{array}
$$

where $\xi \in[0,1]$ is a parameter. For $\xi>\frac{1}{2}$, these interface conditions can be rewritten as follows,

$$
\begin{array}{ll}
\eta_{\Gamma} \llbracket \mathbf{u} \cdot \mathbf{n}_{\Gamma} \rrbracket=\frac{4}{2 \xi-1}(\{p\}-\hat{p}) & \text { on } \Gamma, \\
\eta_{\Gamma}\left\{\mathbf{u} \cdot \mathbf{n}_{\Gamma}\right\}=\llbracket p \rrbracket & \text { on } \Gamma .
\end{array}
$$

Note that (2.2) are recovered from (2.4) in the limit $\xi \rightarrow \frac{1}{2}^{+}$.

The pressure $\hat{p}$ can be known a priori, or it can be computed solving a fracture flow problem. This requires the derivation of a reduced model for the fracture flow, for which we refer to $[1,2,12,15]$. Following the cited references, we assume that the flow within the fracture is governed by a reduced Darcy's equation. Define the tangential gradient $\nabla_{\tau}=\left(\boldsymbol{\tau}_{\Gamma} \boldsymbol{\tau}_{\Gamma}^{T}\right) \nabla$ as the projection of the gradient onto the tangent space. Let $\mathbf{u}_{\Gamma}$ be the fluid velocity in the fracture, and let $\hat{\mathbf{u}}=l_{\Gamma}\left(\boldsymbol{\tau}_{\Gamma} \boldsymbol{\tau}_{\Gamma}^{T}\right) \mathbf{u}_{\Gamma}$ (which is a "mean flow rate" within the fracture, in the tangential direction). Denote by $\hat{\boldsymbol{f}}_{v}$ a generic momentum forcing term and by $\hat{f}_{q}$ a mass source term in the fracture. Although different boundary conditions would be admissible, for the sake of simplicity and symmetry with respect to the boundary conditions of the bulk flow problem, we will split the endpoints $\partial \Gamma$ of $\Gamma$ in a Dirichlet boundary $\partial_{D} \Gamma$, where no flow takes place, and a homogeneous Neumann boundary $\partial_{N} \Gamma$, where the pressure $\hat{p}$ is set to a reference value. In this case, the fracture velocity and pressure satisfy the following problem,

$$
\begin{array}{llll}
\hat{\eta} \hat{\mathbf{u}}+\nabla_{\tau} \hat{p}=\hat{\boldsymbol{f}}_{v} & \text { on } \Gamma, & \hat{\mathbf{u}} \cdot \boldsymbol{\tau}_{\Gamma}=0 & \text { on } \partial_{D} \Gamma, \\
\nabla_{\tau} \cdot \hat{\mathbf{u}}=\hat{f}_{q}(\mathbf{u}) & \text { on } \Gamma, & \hat{p}=\hat{p}_{0} & \text { on } \partial_{N} \Gamma,
\end{array}
$$

where $\hat{\eta}=\left[l_{\Gamma} \mathrm{K}_{\Gamma, \tau}\right]^{-1}$, and $\hat{f}_{q}(\mathbf{u})=l_{\Gamma} \tilde{f}_{q}+\left(\mathbf{u}_{1}-\mathbf{u}_{2}\right) \cdot \mathbf{n}_{\Gamma}$.

Equations (2.1), (2.5), with interface conditions (2.3), constitute a system of coupled problems governing the fluid motion in the fractured domain. In [15], the well posedness of such coupled problem has been proved for $\frac{1}{2}<\xi \leq 1$. We will show in the next section that this assumption is needed also in our formulation. Hence, in general we will refer to coupling conditions (2.4) with $\frac{1}{2}<\xi \leq 1$.

In the next sections, we will proceed along the following lines. In Sections 3 and 4 we consider the problem of the numerical approximation of (2.1), (2.4) with an unfitted mixed finite element scheme, assuming that $\hat{p}$ is 
known, focusing on the treatment of fractures which are not conformal with the triangulation on $\Omega$, studying the algebraic properties of the discrete problem. In Section 5 we introduce an iterative scheme to include the fracture flow equations and solve the coupled problems. Finally, we will verify the predicted properties by numerical experiments.

\section{Finite element approximation of the BUlK Flow Problem}

In order to set up our finite element scheme, let us assume that $\Omega$ is a convex polygon, and consider a family of triangulations $\mathcal{T}_{h}$, i.e. collections of simplexes (triangles or tetrahedra in 2D/3D), being $h$ the maximal diameter of the elements of $\mathcal{T}_{h}$. We point out that $\mathcal{T}_{h}$ may not be conformal with the fracture $\Gamma$, so that triangles $K \in \mathcal{T}_{h}$ may be cut by $\Gamma$.

The technique of enriching the elements cut by an "embedded" interface with discontinuous functions is the basic idea of the extended finite element method [16], originally developed for the computational analysis of the evolution of cracks in solid mechanics. Recently, this idea has been applied in combination with Nitsche's method based on penalization, see [6,14]. Here we follow a similar approach considering Raviart-Thomas finite elements and coupled porous domain/fracture domain problems.

In this work the notation $a \lesssim b$ means that there is a constant $C>0$, independent of $h$ and of the physical parameters $\eta, \hat{\eta}, \eta_{\Gamma}$, such that $a \leq C b$; we will use $a \gtrsim b$ similarly. Following [14], we require that the following assumptions are satisfied.

A1. The triangulation is shape-regular, i.e., $\rho_{K} \lesssim h_{K} \lesssim \rho_{K} \forall K \in \mathcal{T}_{h}$, where $h_{K}$ is the diameter of $K$ and $\rho_{K}$ is the diameter of the largest ball contained in $K$.

A2. If $\Gamma \cap K \neq \emptyset, K \in \mathcal{T}_{h}$, then $\Gamma$ intersects $\partial K$ exactly twice, and each (open) edge at most once.

A3. Let $\Gamma_{K, h}$ be the straight line segment connecting the points of intersection between $\Gamma$ and $\partial K$. We assume that $\Gamma_{K}$ is a function of length on $\Gamma_{K, h}$ : in particular, in local coordinates $(s, t)$ we have $\Gamma_{K, h}=\{(s, t): 0<$ $\left.s<\left|\Gamma_{K, h}\right|, t=0\right\}$ and $\Gamma_{K}=\left\{(s, t): 0<s<\left|\Gamma_{K, h}\right|, t=\delta(s)\right\}$, where $\delta$ is positive in the direction of $\mathbf{n}_{\Gamma}$, i.e., $\mathbf{n}_{\Gamma}=\frac{\left(-\delta^{\prime}(s), 1\right)^{T}}{\left(\delta^{\prime}(s)^{2}+1\right)^{1 / 2}}$ in local coordinates. This hypothesis is always fulfilled on sufficiently fine meshes if $\Gamma$ has bounded curvature.

We shall denote $K_{i}=K \cap \Omega_{i}$ for any element $K \in \mathcal{T}_{h}$, and $\mathcal{G}_{h}=\left\{K \in \mathcal{T}_{h}: \Gamma \cap K \neq \emptyset\right\}$ the collection of elements that are crossed by the fracture. Let us introduce the finite element spaces that will be used in the set up of the method.

For any $K \in \mathcal{T}_{h}$, let $\mathbb{R T}_{0}\left(K_{i}\right)=\left\{\mathbf{v}_{h_{K_{i}}}: \mathbf{v}_{h} \in \mathbb{R T}_{0}(K)\right\}$ be the linear space of the restrictions to $K_{i}$ of the standard $\mathbb{R}_{0}$ local functions; define analogously $\mathbb{P}_{0}\left(K_{i}\right)$.

We consider discrete velocities $\mathbf{v}_{h}$ and pressures $q_{h}$ in the following spaces,

$$
\begin{aligned}
& \mathbf{V}_{h}=\mathbf{V}_{1, h} \times \mathbf{V}_{2, h}, \quad Q_{h}=Q_{1, h} \times Q_{2, h}, \\
\mathbf{V}_{i, h}= & \left\{\mathbf{v}_{h} \in \boldsymbol{H}_{\text {div }}\left(\Omega_{i}\right): \mathbf{v}_{h_{K_{i}}} \in \mathbb{R T}_{0}\left(K_{i}\right) \forall K \in \mathcal{T}_{h}\right\}, \\
Q_{i, h}= & \left\{q_{h} \in L^{2}\left(\Omega_{i}\right): q_{\left.\right|_{K_{i}}} \in \mathbb{P}_{0}\left(K_{i}\right) \forall K \in \mathcal{T}_{h}\right\} .
\end{aligned}
$$

Each discrete velocity $\mathbf{v}_{h}=\left(\mathbf{v}_{1, h}, \mathbf{v}_{2, h}\right)$ and pressure $q_{h}=\left(q_{1, h}, q_{2, h}\right)$ is thus made of two components, associated to the domains $\Omega_{i}, i=1,2$. The discrete variables are discontinuous on $\Gamma$, being defined on each part $K_{i}$ of a cut element $K \in \mathcal{G}_{h}$ by indipendent $\left(\mathbb{R}_{0}, \mathbb{P}_{0}\right)$ local functions. In other words, on cut elements each local function is actually a pair of indipendent restrictions of the traditional finite element spaces to the two sub-regions.

The finite element basis for the spaces $\mathbf{V}_{h}$ and $Q_{h}$ are obtained, following the approach proposed in [14], from the standard $\mathbb{R}_{0}$ and $\mathbb{P}_{0}$ basis on the mesh replacing each standard basis function living on an element that intersects the interface by its restrictions to $\Omega_{1}$ and $\Omega_{2}$ respectively, as shown in Figure 1. 


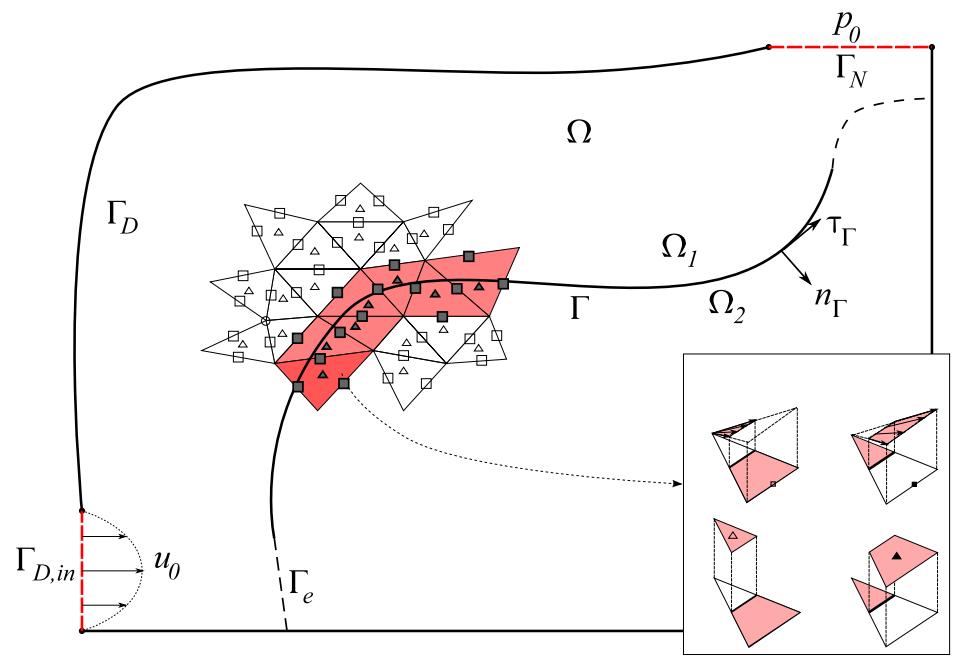

Figure 1. The porous domain $\Omega$ and the fracture $\Gamma$. The boundary $\partial \Omega=\Gamma_{D} \cup \Gamma_{N}$ is split in a Dirichlet boundary (where the Darcy's normal velocity is imposed) and a Neumann boundary (where the pressure is prescribed). The inflow $\Gamma_{D, i n} \subset \Gamma_{D}$ is also shown (homogeneous conditions being prescribed on $\left.\Gamma_{D} \backslash \Gamma_{D, \text { in }}\right)$. Standard $\mathbb{P}_{0}$ degrees of freedom of the pressure associated to internal nodes (marked with black triangles) are duplicated (gray triangles) on elements $K \in \mathcal{G}_{h}$ crossed by $\Gamma$ (shaded), to provide constant pressure on each sub-element $K_{1}$, $K_{2}$. Analogously, the $\mathbb{R T}_{0}$ degrees of freedom of the velocity, associated to the edges midpoints (black squares) are duplicated (gray squares) on elements $K \in \mathcal{G}_{h}$, leading to independent $\mathbb{R T}_{0}$ functions on $K_{1}$ and $K_{2}$, as outlined in the box. Note also that a "partially immersed fracture" $\Gamma$ can be treated as a full interface $\Gamma \cup \Gamma_{e}$ between $\Omega_{1}$ and $\Omega_{2}$, and imposing the continuity of the pressure and of the normal velocity on $\Gamma_{e}$ by properly modifying the interface conditions (2.4).

For the sake of simplicity, we shall consider the case in which $\eta$ is a scalar rather than a positive definite tensor; to further ease the analysis, we will assume that $\eta=\eta_{i} \in \mathbb{R}$ on each subdomain $\Omega_{i}$. The numerical scheme can be easily modified to account for variable, tensor valued coefficients.

Let us introduce the following bilinear and linear forms:

$$
\begin{aligned}
a\left(\mathbf{u}_{h}, \mathbf{v}_{h}\right)= & \int_{\Omega} \eta \mathbf{u}_{h} \cdot \mathbf{v}_{h}+\int_{\Gamma_{D}} \gamma h^{-1}\left(\mathbf{u}_{h} \cdot \mathbf{n}\right)\left(\mathbf{v}_{h} \cdot \mathbf{n}\right) \\
& +\int_{\Gamma} \eta_{\Gamma}\left\{\mathbf{u}_{h} \cdot \mathbf{n}_{\Gamma}\right\}\left\{\mathbf{v}_{h} \cdot \mathbf{n}_{\Gamma}\right\}+\xi_{0} \int_{\Gamma} \eta_{\Gamma} \llbracket \mathbf{u}_{h} \cdot \mathbf{n}_{\Gamma} \rrbracket \llbracket \mathbf{v}_{h} \cdot \mathbf{n}_{\Gamma} \rrbracket, \\
b\left(p_{h}, \mathbf{v}_{h}\right)= & -\int_{\Omega} p_{h}\left(\nabla \cdot \mathbf{v}_{h}\right)+\int_{\Gamma_{D}} p_{h}\left(\mathbf{v}_{h} \cdot \mathbf{n}\right), \\
\mathcal{F}\left(\mathbf{v}_{h}, q_{h}\right)= & \int_{\Omega} \boldsymbol{f}_{v} \cdot \mathbf{v}_{h}-\int_{\Gamma_{N}} p_{0}\left(\mathbf{v}_{h} \cdot \mathbf{n}\right)+\int_{\Gamma_{D}} \gamma h^{-1} u_{0}\left(\mathbf{v}_{h} \cdot \mathbf{n}\right) \\
& +\int_{\Omega} f_{q} q_{h}-\int_{\Gamma_{D}} u_{0} q_{h}-\int_{\Gamma} \hat{p} \llbracket \mathbf{v}_{h} \cdot \mathbf{n}_{\Gamma} \rrbracket,
\end{aligned}
$$

where $\gamma$ is a positive penalization coefficient, $2 \xi_{0}=\xi-\frac{1}{2}$, and where we denote (with a little abuse of notation) $h$ a piecewise constant function defined on all edges $E \subset \partial K, K \in \mathcal{T}_{h}$, and such that $h_{\mid E}=\operatorname{diam}(E)$. Note that in order to ensure the strict positivity of the associated interface term, the assumption $\xi>\frac{1}{2}$ is indeed required, even if our formulation also accommodates the limit case $\xi=\frac{1}{2}$ (or $\left.\xi_{0}=0\right)$. 
Our finite element method reads as follows: given the boundary data $u_{0}, p_{0}$, and the fracture pressure $\hat{p}$, find $\left(\mathbf{u}_{h}, p_{h}\right) \in \mathbf{W}_{h}$ such that

$$
\begin{aligned}
& \mathcal{C}\left(\left(\mathbf{u}_{h}, p_{h}\right),\left(\mathbf{v}_{h}, q_{h}\right)\right)=\mathcal{F}\left(\mathbf{v}_{h}, q_{h}\right) \quad \forall\left(\mathbf{v}_{h}, q_{h}\right) \in \mathbf{W}_{h}, \\
& \text { where } \\
& \mathcal{C}\left(\left(\mathbf{u}_{h}, p_{h}\right),\left(\mathbf{v}_{h}, q_{h}\right)\right)=a\left(\mathbf{u}_{h}, \mathbf{v}_{h}\right)+b\left(p_{h}, \mathbf{v}_{h}\right)-b\left(q_{h}, \mathbf{u}_{h}\right) .
\end{aligned}
$$

The choice of the $\left(\mathbb{R}_{0}, \mathbb{P}_{0}\right)$ finite element pair is typical for applications to geophysical problems. Single and possibly multi-phase flows in porous media require robust, locally conservative numerical method to provide accurate solution without mass losses. We also point out that different formulations using stabilized $\left(\mathbb{P}_{k+1}, \mathbb{P}_{k}\right)$ finite elements (with interior penalties) for the Darcy's equation have been studied in $[9,10]$, which allow a unified formulation in the framework of coupling with viscous flows. On a similar finite element pair is also based the work presented in [6]. In such works, it has been shown that Nitsche's method is a flexible tool to treat interface conditions (IC). We observe that ICs (2.4) are of mixed (Robin) type, relating velocity to stress (pressure); no essential IC is considered. Correspondingly, in this work we insert (2.4) as natural ICs in our variational formulation. However, we make use of the Nitsche's method to impose the Dirichlet boundary conditions. Different formulations could extend the application of the method also for the ICs.

\section{Consistency, Stability And COnVERGEnCE AnAlysis}

Under minimal regularity assumptions, the finite element scheme (3.4) is consistent with (2.1), (2.4). Let us introduce the following spaces

$$
\begin{aligned}
& \mathbf{V}=\left\{\mathbf{v}=\left(\mathbf{v}_{1}, \mathbf{v}_{2}\right): \mathbf{v}_{i} \in \boldsymbol{H}_{\operatorname{div}}\left(\Omega_{i}\right), \mathbf{v}_{i} \cdot \mathbf{n}_{\Gamma} \in L^{2}(\Gamma), i=1,2\right\}, \\
& Q=\left\{q=\left(q_{1}, q_{2}\right): q_{i} \in H^{1}\left(\Omega_{i}\right)\right\} \subset L^{2}(\Omega), \quad \mathbf{W}=\mathbf{V} \times Q .
\end{aligned}
$$

Note that the normal component $\mathbf{v} \cdot \mathbf{n}_{\Gamma}$ of a function $\mathbf{v} \in \mathbf{V}$ is discontinuous on $\Gamma$.

Lemma 4.1 (consistency). Let $(\mathbf{u}, p)$ be the solution of (2.1) with interface conditions (2.4), and let $\left(\mathbf{u}_{h}, p_{h}\right) \in$ $\mathbf{W}_{h}$ be the solution of (3.4). If $(\mathbf{u}, p) \in \mathbf{W}$, we have

$$
\mathcal{C}\left(\left(\mathbf{u}-\mathbf{u}_{h}, p-p_{h}\right),\left(\mathbf{v}_{h}, q_{h}\right)\right)=0 \quad \forall\left(\mathbf{v}_{h}, q_{h}\right) \in \mathbf{W}_{h} .
$$

Proof. Let us show that $\mathcal{C}\left((\mathbf{u}, p),\left(\mathbf{v}_{h}, q_{h}\right)\right)=\mathcal{F}\left(\mathbf{v}_{h}, q_{h}\right) \forall\left(\mathbf{v}_{h}, q_{h}\right) \in \mathbf{W}_{h}$. Using the Green's theorem in both subdomains $^{3} \Omega_{i}, i=1,2$, we have $b\left(p, \mathbf{v}_{h}\right)=\int_{\Omega} \mathbf{v}_{h} \cdot \nabla p-\int_{\Gamma_{N}} p\left(\mathbf{v}_{h} \cdot \mathbf{n}\right)-\int_{\Gamma} \llbracket p\left(\mathbf{v}_{h} \cdot \mathbf{n}_{\Gamma}\right) \rrbracket$. Hence, replacing $\eta \mathbf{u}+\nabla p=\boldsymbol{f}_{v}, \nabla \cdot \mathbf{u}=f_{q}$, and using the boundary conditions, we have

$$
\begin{aligned}
\mathcal{C}\left((\mathbf{u}, p),\left(\mathbf{v}_{h}, q_{h}\right)\right)= & \int_{\Omega} \boldsymbol{f}_{v} \cdot \mathbf{v}_{h}+\int_{\Gamma} \eta_{\Gamma}\left\{\mathbf{u} \cdot \mathbf{n}_{\Gamma}\right\}\left\{\mathbf{v}_{h} \cdot \mathbf{n}_{\Gamma}\right\}+\xi_{0} \int_{\Gamma} \eta_{\Gamma} \llbracket \mathbf{u}_{h} \cdot \mathbf{n}_{\Gamma} \rrbracket \llbracket \mathbf{v}_{h} \cdot \mathbf{n}_{\Gamma} \rrbracket \\
& +\int_{\Gamma_{D}} \gamma h^{-1} u_{0}\left(\mathbf{v}_{h} \cdot \mathbf{n}\right)-\int_{\Gamma_{N}} p_{0}\left(\mathbf{v}_{h} \cdot \mathbf{n}\right)-\int_{\Gamma} \llbracket p\left(\mathbf{v}_{h} \cdot \mathbf{n}_{\Gamma}\right) \rrbracket+\int_{\Omega} f_{q} \nabla \cdot \mathbf{v}_{h}-\int_{\Gamma_{D}} q_{h} u_{0} .
\end{aligned}
$$

Thanks to the algebraic identity $\llbracket a b \rrbracket=\llbracket a \rrbracket\{b\}+\{a\} \llbracket b \rrbracket$ and to the interface conditions (2.4) we can write

$$
\int_{\Gamma} \llbracket p\left(\mathbf{v}_{h} \cdot \mathbf{n}_{\Gamma}\right) \rrbracket=\int_{\Gamma} \llbracket p \rrbracket\left\{\mathbf{v}_{h} \cdot \mathbf{n}_{\Gamma}\right\}+\int_{\Gamma}\{p\} \llbracket \mathbf{v}_{h} \cdot \mathbf{n}_{\Gamma} \rrbracket=I+I I
$$

where

$$
I=\int_{\Gamma} \eta_{\Gamma}\left\{\mathbf{u} \cdot \mathbf{n}_{\Gamma}\right\}\left\{\mathbf{v}_{h} \cdot \mathbf{n}_{\Gamma}\right\}, \quad I I=\xi_{0} \int_{\Gamma} \eta_{\Gamma} \llbracket \mathbf{u} \cdot \mathbf{n}_{\Gamma} \rrbracket \llbracket \mathbf{v}_{h} \cdot \mathbf{n}_{\Gamma} \rrbracket+\int_{\Gamma} \hat{p} \llbracket \mathbf{v}_{h} \cdot \mathbf{n}_{\Gamma} \rrbracket,
$$

\footnotetext{
${ }^{3}$ In the case of a "partially immersed" fracture this step would be more complex, see [2], Section 3.
} 
so that

$$
\begin{aligned}
\mathcal{C}\left((\mathbf{u}, p),\left(\mathbf{v}_{h}, q_{h}\right)\right)= & \int_{\Omega} \boldsymbol{f}_{v} \cdot \mathbf{v}_{h}+\int_{\Gamma_{D}} \gamma h^{-1} u_{0}\left(\mathbf{v}_{h} \cdot \mathbf{n}\right)-\int_{\Gamma_{N}} p_{0}\left(\mathbf{v}_{h} \cdot \mathbf{n}\right)+\int_{\Omega} f_{q} \nabla \cdot \mathbf{v}_{h} \\
& -\int_{\Gamma} \hat{p} \llbracket \mathbf{v}_{h} \cdot \mathbf{n}_{\Gamma} \rrbracket-\int_{\Gamma_{D}} q_{h} u_{0}=\mathcal{F}\left(\mathbf{v}_{h}, q_{h}\right) .
\end{aligned}
$$

We will consider the following discrete norms:

$$
\begin{aligned}
\left.\left\|\mathbf{v}_{h}\right\|\right|^{2} & =\left\|\eta^{\frac{1}{2}} \mathbf{v}_{h}\right\|_{L^{2}(\Omega)}^{2}+\left\|\eta_{\Gamma}^{\frac{1}{2}}\left\{\mathbf{v}_{h} \cdot \mathbf{n}_{\Gamma}\right\}\right\|_{L^{2}(\Gamma)}^{2}+\xi_{0}\left\|\eta_{\Gamma}^{\frac{1}{2}} \llbracket \mathbf{v}_{h} \cdot \mathbf{n}_{\Gamma} \rrbracket\right\|_{L^{2}(\Gamma)}^{2}+\left\|h^{-\frac{1}{2}} \mathbf{v}_{h} \cdot \mathbf{n}\right\|_{L^{2}\left(\Gamma_{D}\right)}^{2}, \\
\left\|\mathbf{v}_{h}\right\|_{\mathbf{V}_{h}}^{2} & =\left\|\mathbf{v}_{h}\right\|\left\|^{2}+\right\| \eta^{\frac{1}{2}} \nabla \cdot \mathbf{v}_{h} \|_{L^{2}(\Omega)}^{2}, \\
\left\|q_{h}\right\|_{Q_{h}}^{2} & =\left\|\eta^{-\frac{1}{2}} q_{h}\right\|_{L^{2}(\Omega)}^{2},
\end{aligned}
$$

and define

$$
\left\|\left(\mathbf{v}_{h}, q_{h}\right)\right\|_{\mathbf{W}_{h}}^{2}=\left\|\mathbf{v}_{h}\right\|_{\mathbf{v}_{h}}^{2}+\left\|q_{h}\right\|_{Q_{h}}^{2}
$$

Note that $\left\|\eta^{\frac{1}{2}} \nabla \cdot \mathbf{v}_{h}\right\|_{L^{2}(\Omega)}^{2}$ is in fact a broken norm, since $\eta^{\frac{1}{2}} \nabla \cdot \mathbf{v}_{h}$ stands for $\left(\eta_{1}^{\frac{1}{2}} \nabla \cdot \mathbf{v}_{1, h}, \eta_{2}^{\frac{1}{2}} \nabla \cdot \mathbf{v}_{2, h}\right)$; however, it is sometimes convenient to identify $L^{2}\left(\Omega_{1}\right) \times L^{2}\left(\Omega_{2}\right)$ with $L^{2}(\Omega)$. Sometimes we will also make use of the $h$-dependent norms $\|u\|_{h, \pm \frac{1}{2}, \Sigma}^{2}:=\int_{\Sigma} h^{\mp 1} u^{2}$.

Lemma $4.2\left(\mathcal{F}\right.$-boundedness). If $\xi_{0}>0$, there exists a constant $C_{h}$ (depending on $h$ and on the quantities reported below) such that

$$
\mathcal{F}\left(\mathbf{v}_{h}, q_{h}\right) \leq C_{h}\left(\boldsymbol{f}_{v}, f_{q}, u_{0}, p_{0} ; \xi_{0}, \hat{p}\right)\left\|\mathbf{v}_{h}, q_{h}\right\|_{\mathbf{W}_{h}} \quad \forall\left(\mathbf{v}_{h}, q_{h}\right) \in \mathbf{W}_{h} .
$$

Proof. We can estimate each term in $\mathcal{F}$ as follows,

$$
\begin{aligned}
\mathcal{F}\left(\mathbf{v}_{h}, q_{h}\right) \leq & \left\|\eta^{-\frac{1}{2}} \boldsymbol{f}_{v}\right\|_{L^{2}(\Omega)}\left\|\eta^{\frac{1}{2}} \mathbf{v}_{h}\right\|_{L^{2}(\Omega)}+\left\|\eta^{-\frac{1}{2}} p_{0}\right\|_{h,-\frac{1}{2}, \Gamma_{N}}\left\|\eta^{\frac{1}{2}} \mathbf{v}_{h} \cdot \mathbf{n}_{\Gamma}\right\|_{h,+\frac{1}{2}, \Gamma_{N}} \\
& +\gamma\left\|u_{0}\right\|_{h,+\frac{1}{2}, \Gamma_{D}}\left\|\mathbf{v}_{h} \cdot \mathbf{n}_{\Gamma}\right\|_{h,+\frac{1}{2}, \Gamma_{D}}+\left\|\eta^{\frac{1}{2}} f_{q}\right\|_{L^{2}(\Omega)}\left\|\eta^{-\frac{1}{2}} q_{h}\right\|_{L^{2}(\Omega)} \\
& +\left\|\eta^{\frac{1}{2}} u_{0}\right\|_{h,+\frac{1}{2}, \Gamma_{D}}\left\|\eta^{-\frac{1}{2}} q_{h}\right\|_{h,-\frac{1}{2}, \Gamma_{D}}+\left\|\eta_{\Gamma}^{-\frac{1}{2}} \hat{p}\right\|_{L^{2}(\Gamma)}\left\|\eta_{\Gamma}^{\frac{1}{2}} \llbracket \mathbf{v}_{h} \cdot \mathbf{n}_{\Gamma} \rrbracket\right\|_{L^{2}(\Gamma)} .
\end{aligned}
$$

Using standard inverse inequalities we have, for $\Sigma=\Gamma_{D}$ or $\Gamma_{N}$,

$$
\left\|\eta^{\frac{1}{2}} \mathbf{v}_{h} \cdot \mathbf{n}_{\Gamma}\right\|_{h,+\frac{1}{2}, \Sigma} \lesssim\left\|\mathbf{v}_{h}\right\|_{\mathbf{v}_{h}}, \quad\left\|\eta^{-\frac{1}{2}} q_{h}\right\|_{h,-\frac{1}{2}, \Sigma} \lesssim\left\|q_{h}\right\|_{Q_{h}} .
$$

By using Cauchy-Schwarz inequality, we get the boundedness result with

$$
\begin{aligned}
C_{h}\left(\boldsymbol{f}_{v}, f_{q}, u_{0}, p_{0} ; \xi_{0}, \hat{p}\right)^{2} \lesssim & \left\|\eta^{-\frac{1}{2}} \boldsymbol{f}_{v}\right\|_{L^{2}(\Omega)}^{2}+\left\|\eta^{-\frac{1}{2}} p_{0}\right\|_{h,-\frac{1}{2}, \Gamma_{N}}^{2}+\gamma^{2}\left\|u_{0}\right\|_{h,+\frac{1}{2}, \Gamma_{D}}^{2} \\
& +\left\|\eta^{\frac{1}{2}} f_{q}\right\|_{L^{2}(\Omega)}^{2}+\left\|\eta^{\frac{1}{2}} u_{0}\right\|_{h,+\frac{1}{2}, \Gamma_{D}}^{2}+\xi_{0}^{-1}\left\|\eta_{\Gamma}^{-\frac{1}{2}} \hat{p}\right\|_{L^{2}(\Gamma)}^{2}
\end{aligned}
$$

Note that for $\xi_{0} \rightarrow 0$ the linear functional $\mathcal{F}$ becomes unbounded (we loose the control of the jump of the normal velocity across the fracture). The dependence on $h$ concerns only the non-homogeneous boundary data $p_{0}$ and $u_{0}$, and will not affect the convergence properties of our scheme when estimating the error.

Similarly, the following properties are immediately verified.

Lemma 4.3 (boundedness). The bilinear form $\mathcal{C}$ is bounded, i.e.

$$
\mathcal{C}\left(\left(\mathbf{u}_{h}, p_{h}\right),\left(\mathbf{v}_{h}, q_{h}\right)\right) \leq\left\|\mathbf{u}_{h}, p_{h}\right\|_{\mathbf{w}_{h}}\left\|\mathbf{v}_{h}, q_{h}\right\|_{\mathbf{w}_{h}} \quad \forall\left(\mathbf{u}_{h}, p_{h}\right),\left(\mathbf{v}_{h}, q_{h}\right) \in \mathbf{W}_{h} .
$$



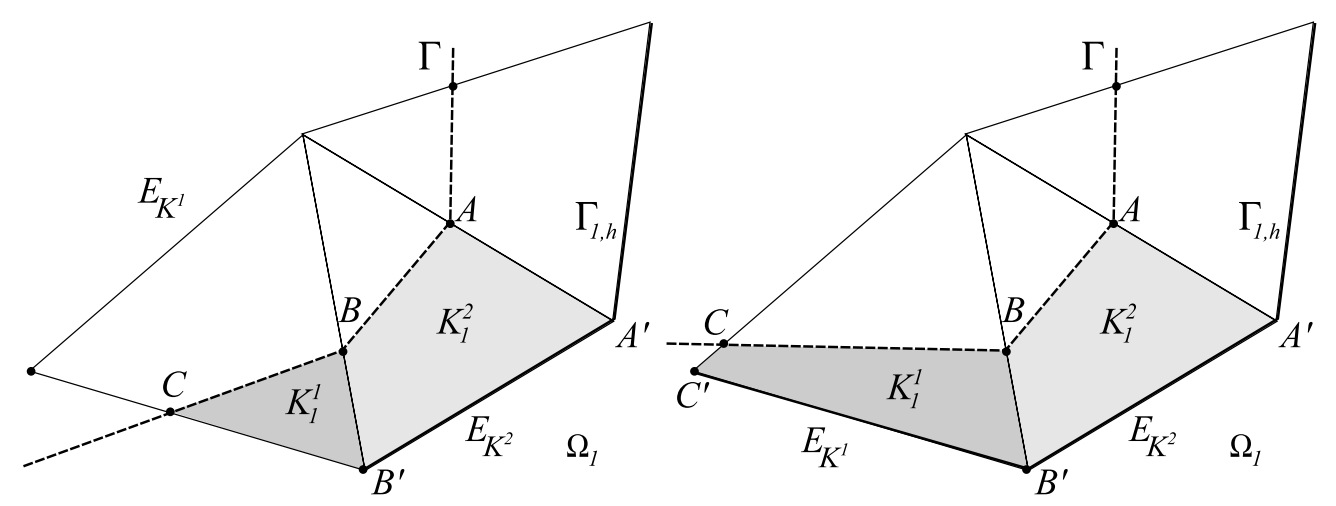

Figure 2. Two elements $K^{j} \in \mathcal{G}_{h}, j=1,2$, cut by an interface $\Gamma=A B C$. On the left: $K^{1} \sim T$ ( $K_{1}^{1}$ is a triangle, $E_{K^{1}}$ is outside $\Omega_{1}$ ), $K^{2} \sim Q\left(K_{1}^{2}\right.$ is a quadrangle, $E_{K^{2}}$ is on $\left.\Gamma_{1, h} \subset \Omega_{1}\right)$. On the right: $K^{1}, K^{2} \sim Q$ ( $K_{1}^{j}$ is a quadrangle, $E_{K^{j}}$ is on $\left.\Gamma_{1, h} \subset \Omega_{i}, j=1,2\right)$.

Lemma 4.4 (positivity). Provided that $\gamma \gtrsim 1$,

$$
\mathcal{C}\left(\left(\mathbf{v}_{h}, q_{h}\right),\left(\mathbf{v}_{h}, q_{h}\right)\right) \geq\left\|\left|\mathbf{v}_{h} \|\right|^{2} \quad \forall\left(\mathbf{v}_{h}, q_{h}\right) \in \mathbf{W}_{h} .\right.
$$

Proof. We have

$$
\mathcal{C}\left(\left(\mathbf{v}_{h}, q_{h}\right),\left(\mathbf{v}_{h}, q_{h}\right)\right)=a\left(\mathbf{v}_{h}, \mathbf{v}_{h}\right)=\left\|\left|\mathbf{v}_{h}\right|\right\|^{2}
$$

so that the lemma follows immediately.

In [6] an "extended" Clément interpolant has been constructed to derive a stabilized inf-sup condition for the Stokes (elasticity) problem using the $\left(\mathbb{P}_{1}, \mathbb{P}_{0}\right)$ finite element pair; the same scheme can be used for the Darcy's problem, see $[9,10]$. In [19] it was numerically shown that if a inf-sup stable, mixed finite element scheme is extended in the pressure space only, degeneration of the inf-sup constant may occur (and can be cured by a suitable modification of the extended space).

Here we prove that using the extended $\left(\mathbb{R T}_{0}, \mathbb{P}_{0}\right)$ pair we can obtain an inf-sup condition under some additional assumptions on the interface $\Gamma$. First of all, we split the domain $\Omega$ in three subregions. One is the strip of (cut) elements in $\mathcal{G}_{h}$; then, we also define $\mathcal{T}_{i, h}=\left\{K \in \mathcal{T}_{h}: K \subset \Omega_{i}\right\}$ the collections of all the elements fully included in each $\Omega_{i}$. We shall refer to the subregions formed by all such elements using the same symbols. Thus, $\Omega=\mathcal{T}_{1, h} \cup \mathcal{G}_{h} \cup \mathcal{T}_{2, h}$ is a disjoint union. We denote by $\Gamma_{i, h}$ the interface between $\mathcal{T}_{i, h}$ and $\mathcal{G}_{h}$.

For each $K \in \mathcal{G}_{h}$, let $E_{K}$ be the only edge of $K$ that is not cut by $\Gamma$. For a given index $i=1,2$, there are only two cases: either $E_{K} \cap \Omega_{i}=\emptyset$, or $E_{K} \subset \Gamma_{i, h}$. In the first case, the sub-element $K_{i}$ is a (curved) triangle: we say that $K$ is of type $T$ and write $K \sim T$. In the other case, the sub-element $K_{i}$ is a (curved) quadrangle: we say that $K$ is of type $Q$ and write $K \sim Q$ (see Fig. 2). Note that this classification depends on $i$.

For each $i=1,2$, it is always possible to split $\mathcal{G}_{h}$ in patches of elements of different types, that we call $P_{n}$ (see Fig. 3 in the case $i=1$ for an example).

- Patches of type $P_{0}$ are formed by a single element $K \sim T$.

- Patches of type $P_{n}$ are formed by one element $K^{\prime} \sim T$ and $n$ elements $K^{j} \sim Q$.

Hypothesis 4.5. Let $K^{1}, K^{2}$ be two adjacent elements in $\mathcal{G}_{h}$ (i.e. sharing an edge $E$ ). There exist two constants $c, C>0$, dependent on $\Gamma$ only, such that

$$
\begin{array}{rr}
\left|K_{i}^{1}\right| \leq C\left|K_{i}^{2}\right| & \text { if } K^{1} \sim T \text { and } K^{2} \sim Q ; \\
c\left|K_{i}^{1}\right| \leq\left|K_{i}^{2}\right| \leq C\left|K_{i}^{1}\right| & \text { if } K^{1}, K^{2} \sim Q .
\end{array}
$$




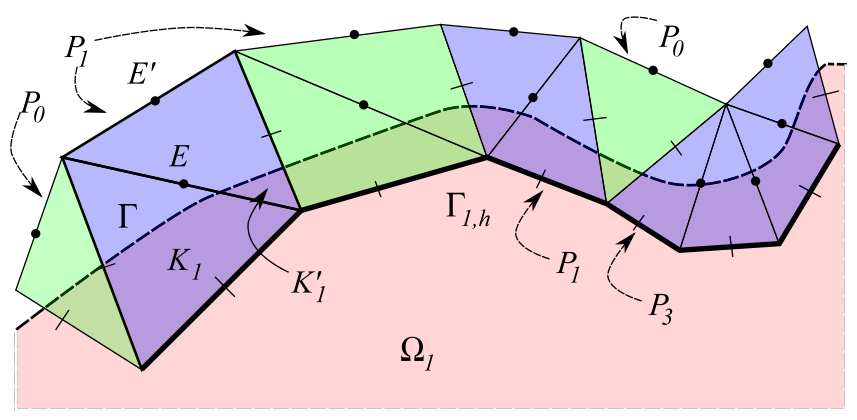

Figure 3 . The strip $\mathcal{G}_{h}$ of all elements cut by $\Gamma$ is split in patches of different types: singleelement $P_{0}$ patches and $n$-elements $P_{n}$ patches. On the restriction to $\Omega_{i}$ of each patch, a local discrete velocity $\mathbf{v}_{i, P_{j}, h}$ is introduced by activating the $\mathbb{R T}_{0}$ degrees of freedom indicated by the circles on the edges. The remaining (inactive) degrees of freedom are denoted by bars: on such edges $\mathbf{v}_{i, P_{j}, h} \cdot \mathbf{n}=0$. Marked are the interface $\Gamma_{1, h}$ between $\mathcal{G}_{h}$ and $\mathcal{T}_{i, h}\left(\mathcal{T}_{i, h}\right.$ being the collection of all elements internal to $\Omega_{i}$ ).

Property 4.6. Hypothesis 4.5 is satisfied if the ratio between the curvature radius $\rho$ of $\Gamma$ and the mesh size $h$ is large enough, i.e. $\rho \geq C_{\Gamma} h$ with $C_{\Gamma}$ a positive constant. Hence, it holds for a sufficiently refined mesh.

Proof. We will restrict the proof to the case in which $\Gamma$ is piecewise linear, as $\Gamma=A B C$ in Figure 2. In this case $\rho$ will be the radius of the circle passing by three consecutive nodes of $\Gamma, \rho=\rho(A, B, C)$. Note also that $\rho(A, B, C) \geq C_{\Gamma} h$ means that $\Gamma$ cannot change direction too rapidly from $A B$ in element $K^{2}$ to $B C$ in element $K^{1}$ - precisely, the angle $A \widehat{B} C$ has to be large enough with respect to the internal angles of the elements, i.e. $A \widehat{B} C \geq \widehat{C}_{\Gamma}$ with $\widehat{C}_{\Gamma}$ a reference angle, that in $2 \mathrm{D}$ can be for instance chosen in $(2 \pi / 3, \pi)$, representing the "typical" mesh curvature, such that $\widehat{C}_{\Gamma} \simeq A^{\prime} \widehat{B}^{\prime} C^{\prime}$ in Figure 2 .

Consider the estimate (4.5): the situation is depicted in Figure 2, on the left. $K_{i}^{1}=B B^{\prime} C \sim T$ is a triangle, and $\left|K_{i}^{1}\right| \lesssim B B^{\prime} \cdot B C$. On the other hand, $K_{i}^{2}=A A^{\prime} B B^{\prime}$ is a quadrangle having a fixed edge $E_{K^{j}} \subset \Gamma_{i, h}$, so that $\left|K_{i}^{2}\right|=\mathcal{O}\left(\left|E_{K^{2}}\right| \cdot \frac{1}{2}\left(B B^{\prime}+A A^{\prime}\right)\right)$. Thanks to shape regularity, $B C \lesssim h \lesssim E_{K^{2}}$, so that (4.5) follows (with no assumptions on $\rho)$.

Now consider (4.6): the situation is depicted in Figure 2, on the right. $K_{i}^{1}=B B^{\prime} C C^{\prime} \sim Q$ and $K_{i}^{2}=$ $A A^{\prime} B B^{\prime} \sim Q$ are both quadrangles. Let us show that $\left|K_{i}^{1}\right| \gtrsim\left|K_{i}^{2}\right|$ (the opposite estimate follows by symmetry). We have $\left|K_{i}^{1}\right|=\mathcal{O}\left(\left|E_{K^{1}}\right| \cdot \frac{1}{2}\left(B B^{\prime}+C C^{\prime}\right)\right),\left|K_{i}^{2}\right|=\mathcal{O}\left(\left|E_{K^{2}}\right| \cdot \frac{1}{2}\left(B B^{\prime}+A A^{\prime}\right)\right)$. Thanks to shape regularity, $E_{K^{2}} \lesssim E_{K^{1}} \lesssim E_{K^{2}}$. To conclude, we need to show that $\frac{1}{2}\left(B B^{\prime}+C C^{\prime}\right)$ cannot degenerate w.r.t. $\frac{1}{2}\left(B B^{\prime}+A A^{\prime}\right)$. This can only happen if $A$ is kept fixed and $B, C$ tend respectively to $B^{\prime}, C^{\prime}$, so that $\left|K_{1}^{1}\right| /\left|K_{1}^{2}\right|$ is arbitrarily small, while $K^{1}$ and $K^{2}$ are both of type $Q$. Note that this implies $A \widehat{B} C<A^{\prime} \widehat{B}^{\prime} C^{\prime}$, i.e. $A \widehat{B} C<\widehat{C}_{\Gamma}$. As we said, that would not be possible if $\rho / h$ is large enough.

As a consequence of (4.5) and (4.6), on each patch $\left\{K^{j} \sim Q, K^{\prime} \sim T, j=1, \ldots, n\right\}$ of type $P_{n}$, we have

$$
\left|K_{i}^{\prime}\right| \lesssim\left|K_{i}^{j}\right|, \quad\left|K_{i}^{j_{2}}\right| \lesssim\left|K_{i}^{j_{1}}\right| \lesssim\left|K_{i}^{j_{2}}\right|, \quad j, j_{1}, j_{2}=1, \ldots, n
$$

We will also need the following auxiliary lemma about the surjectivity of the divergence operator onto $L^{2}$.

Lemma 4.7. Let $\Omega$ be a Lipschitz domain, and let $\Gamma \subset \partial \Omega, \Gamma \neq \partial \Omega$, be a Lipschitz subset of its boundary. For any $q \in L^{2}(\Omega)$, there exists $\mathbf{v} \in \boldsymbol{H}^{1}(\Omega)$ such that

$$
\nabla \cdot \mathbf{v}=q, \quad \mathbf{v}_{\mid \Gamma}=\mathbf{0}, \quad\|\mathbf{v}\|_{H^{1}(\Omega)} \lesssim\|q\|_{L^{2}(\Omega)} .
$$


Proof. If $q \in L_{0}^{2}(\Omega)=\left\{f \in L^{2}(\Omega): \int_{\Omega} f=0\right\}$ and $\Gamma=\partial \Omega$, this is a well known result [13]. To deal with the general case in which $\Gamma$ is only part of the boundary, consider any lifting $\mathbf{h} \in \boldsymbol{H}^{1}(\Omega)$ of a smooth boundary datum satisfying $\mathbf{h}_{\mid \Gamma}=\mathbf{0}, \int_{\partial \Omega} \mathbf{h} \cdot \mathbf{n} \neq 0$. By normalization, we can then require $\int_{\Omega} \nabla \cdot \mathbf{h}=1$. Let $\bar{q}=\int_{\Omega} q \in \mathbb{R}$, and consider $q_{0}=q-\bar{q}(\nabla \cdot \mathbf{h})$. We have $q_{0} \in L_{0}^{2}(\Omega)$. Then, there exists $\mathbf{v}_{0} \in \boldsymbol{H}^{1}(\Omega)$ such that

$$
\nabla \cdot \mathbf{v}_{0}=q_{0}, \quad \mathbf{v}_{0 \mid \partial \Omega}=\mathbf{0}, \quad\left\|\mathbf{v}_{0}\right\|_{H^{1}(\Omega)} \lesssim\left\|q_{0}\right\|_{L^{2}(\Omega)} .
$$

Let $\mathbf{v}=\mathbf{v}_{0}+\bar{q} \mathbf{h}$. Since $\nabla \cdot \mathbf{v}=q_{0}+\bar{q}(\nabla \cdot \mathbf{h})=q$, and $\|\mathbf{v}\|_{H^{1}(\Omega)} \leq\left\|\mathbf{v}_{0}\right\|_{H^{1}(\Omega)}+|\bar{q}|\|\mathbf{h}\|_{H^{1}(\Omega)} \lesssim\left\|q_{0}\right\|_{L^{2}(\Omega)}+|\bar{q}| \lesssim$ $\|q\|_{L^{2}(\Omega)}$ the proof is concluded.

Lemma 4.8 (inf-sup condition). For any $p_{h} \in Q_{h}$, there exists $\mathbf{v}_{p, h} \in \mathbf{V}_{h}$ such that

$$
\begin{aligned}
b\left(p_{h}, \mathbf{v}_{p, h}\right) & \gtrsim\left\|\eta^{-\frac{1}{2}} p_{h}\right\|_{L^{2}(\Omega)}^{2}, \\
\left\|\mathbf{v}_{p, h}\right\|_{\mathbf{v}_{h}} & \lesssim M_{\Gamma}\left\|p_{h}\right\|_{Q_{h}}, \\
\mathbf{v}_{p, h} & =\mathbf{0} \text { on } \Gamma_{D},
\end{aligned}
$$

where $M_{\Gamma}=\max \left\{1, h \sqrt{\eta_{\Gamma} / \eta_{\min }}\right\}, \eta_{\min }=\min \left\{\eta_{1}, \eta_{2}\right\}$.

Proof. Let us analyze the three different subregions $\Omega=\mathcal{T}_{1, h} \cup \mathcal{G}_{h} \cup \mathcal{T}_{2, h}$. We will also need the piecewise constant functions $g_{i, h} \in L^{2}\left(\Omega_{i}\right)$ such that $g_{i, h} K_{i}=\left.\eta_{i}^{-1} p_{i, h}\right|_{\mid K_{i}}$ on each $K$. For the sake of simplicity, we will consider the case in which $\Gamma_{N} \cap \mathcal{T}_{i, h} \neq \emptyset$. Use will be made of standard notations for broken norms, such as $\|v\|_{H^{1}\left(\mathcal{T}_{h}\right)}^{2}=\sum_{K \in \mathcal{T}_{h}}\|v\|_{H^{1}(K)}^{2}$ for the space of (possibly discountinuous) element-wise $H^{1}$ functions on $\mathcal{T}_{h}$.

Let us start with $\mathcal{T}_{i, h} \subset \Omega_{i}$. Thanks to Lemma 4.7, there exists $\overline{\mathbf{v}}_{i} \in \boldsymbol{H}^{1}\left(\Omega_{i}\right)$ such that

$$
\overline{\mathbf{v}}_{i}=\mathbf{0} \text { on } \partial \mathcal{T}_{i, h} \backslash \Gamma_{N}, \quad \nabla \cdot \overline{\mathbf{v}}_{i}=-g_{i, h}, \quad\left\|\overline{\mathbf{v}}_{i}\right\|_{H^{1}\left(\mathcal{T}_{i, h}\right)} \lesssim\left\|g_{i, h}\right\|_{L^{2}\left(\mathcal{T}_{i, h}\right)} .
$$

Note that $\overline{\mathbf{v}}_{i}=\mathbf{0}$ on $\Gamma_{i, h} \subset \partial \mathcal{T}_{i, h}$ and $\left\|\eta_{i}^{\frac{1}{2}} \overline{\mathbf{v}}_{i}\right\|_{H^{1}\left(\mathcal{T}_{i, h}\right)} \lesssim\left\|\eta_{i}^{-\frac{1}{2}} p_{i, h}\right\|_{L^{2}\left(\mathcal{T}_{i, h}\right)}, \eta_{i}$ being constant on $\mathcal{T}_{i, h}$. Owing to the $\boldsymbol{H}_{\text {div }}\left(\mathcal{T}_{i, h}\right)$-conformal $\mathbb{R} \mathbb{T}_{0}$ interpolant $I_{i, h}$ such that $\int_{E}\left(I-I_{i, h}\right) \overline{\mathbf{v}}_{i} \cdot \mathbf{n}_{E}=0$ on all edges $E$ of all elements $K \in \mathcal{T}_{i, h}$, let us define $\overline{\mathbf{v}}_{i, h} \in \mathbf{V}_{h}$ as the extension of $I_{i, h} \overline{\mathbf{v}}_{i}$ by zero on $\Omega \backslash \mathcal{T}_{i, h}$. We have $\left\|\eta_{i}^{\frac{1}{2}} \overline{\mathbf{v}}_{i, h}\right\|_{H^{1}\left(\mathcal{T}_{i, h}\right)} \lesssim\left\|\eta_{i}^{-\frac{1}{2}} p_{i, h}\right\|_{L^{2}\left(\mathcal{T}_{i, h}\right)}$, $\overline{\mathbf{v}}_{i, h} \cdot \mathbf{n}=0$ on $\partial \mathcal{T}_{i, h} \cap \Gamma_{D}$, and

$$
b\left(p_{h}, \overline{\mathbf{v}}_{i, h}\right)=-\int_{\mathcal{T}_{i, h}} p_{i, h}\left(\nabla \cdot \overline{\mathbf{v}}_{i, h}\right)=-\int_{\mathcal{T}_{i, h}} p_{i, h}\left(\nabla \cdot \overline{\mathbf{v}}_{i}\right)=\left\|\eta^{-\frac{1}{2}} p_{h}\right\|_{L^{2}\left(\mathcal{T}_{i, h}\right)}^{2} .
$$

It will now be sufficient to find $\widetilde{\mathbf{v}}_{h} \in \mathbf{V}_{h}$ such that $\operatorname{supp}\left(\widetilde{\mathbf{v}}_{h}\right) \subset \mathcal{G}_{h}, b\left(p_{h}, \widetilde{\mathbf{v}}_{h}\right)=\left\|\eta^{-\frac{1}{2}} p_{h}\right\|_{L^{2}\left(\mathcal{G}_{h}\right)}^{2}$ and $\left\|\eta_{i}^{\frac{1}{2}} \widetilde{\mathbf{v}}_{h}\right\|_{H^{1}\left(\mathcal{G}_{h}\right)} \lesssim\left\|\eta_{i}^{-\frac{1}{2}} p_{i, h}\right\|_{L^{2}\left(\mathcal{G}_{h}\right)}$. We need a special construction since functions in $\mathbf{V}_{h}$ are discountinuous on $\Gamma$

To this end, we exploit the splitting of $\mathcal{G}_{h}$ in patches of element of type $P_{n}$, as follows. Let $K \in \mathcal{G}_{h}$. Note that $p_{h \mid K}$ is represented by a couple of real values $p_{i, K}, i=1,2$, where $p_{i, K}=p_{h \mid K_{i}}$. Recall that $E_{K}$ denotes the only edge of $K$ which is not cut by $\Gamma$.

- If $K \sim T$ is a single element $P_{0}$ patch, define $\widetilde{\mathbf{v}}_{i, K, h} \in \mathbf{V}_{i, h}$ by $\widetilde{\mathbf{v}}_{i, K, h}=\left(m_{E_{K}} \boldsymbol{\phi}_{E_{K}}\right)_{\mid \Omega_{i}}$, where $\phi_{E_{K}}$ is the $\mathbb{R T}_{0}$ basis function associated to $E_{K}$ such that $\phi_{E_{K}} \cdot \mathbf{n}_{K}=1$ on $E_{K}$ and $\phi_{E_{K}} \cdot \mathbf{n}_{K}=0$ on the remaining edges. Such functions are "local" in that the only "active" degree of freedom is not shared with the adjacent elements in $\mathcal{G}_{h}$ (see Fig. 3, where active d.o.f. are represented by small circles on each edge, whereas we have zero normal fluxes on the remaining edges, marked with small bars). The support of such $\widetilde{\mathbf{v}}_{i, K, h}$ is thus $K_{i}$. Let us choose $m_{E_{K}}$ such that $\nabla \cdot \widetilde{\mathbf{v}}_{i, K, h}=-\eta_{i}^{-1} p_{i, K}$. Since on $K$ we have $\nabla \cdot \phi_{E_{K}}=\frac{1}{|K|} \int_{K} \nabla \cdot \phi_{E_{K}}=\frac{1}{|K|} \int_{\partial K} \phi_{E_{K}} \cdot \mathbf{n}_{K}=\frac{\left|E_{K}\right|}{|K|}$, it suffices to set $m_{E_{K}}=-\eta_{i}^{-1} \frac{|K|}{\left|E_{K}\right|} p_{i, K}$, yielding

$$
b\left(p_{h}, \widetilde{\mathbf{v}}_{i, K, h}\right)=-\int_{K_{i}} p_{i, K} \nabla \cdot \widetilde{\mathbf{v}}_{i, K, h}=\int_{K_{i}} \eta_{i}^{-1} p_{i, K}^{2}=\left\|\eta^{-\frac{1}{2}} p_{h}\right\|_{L^{2}\left(K_{i}\right)} .
$$


On the other hand, we have $\left\|\eta_{i}^{\frac{1}{2}} \widetilde{\mathbf{v}}_{i, K, h}\right\|_{H^{1}\left(K_{i}\right)}=\eta_{i}^{\frac{1}{2}}\left|m_{E_{K}}\right|\left\|\phi_{E_{K}}\right\|_{H^{1}\left(K_{i}\right)}$. Owing to standard inverse inequalities, we have $\left\|\phi_{E_{K}}\right\|_{H^{1}\left(K_{i}\right)} \lesssim h^{-1}\left|K_{i}\right|^{\frac{1}{2}}$ and, thanks to shape regularity, $|K| /\left|E_{K}\right| \lesssim h$. Hence,

$$
\left\|\eta_{i}^{\frac{1}{2}} \widetilde{\mathbf{v}}_{i, K, h}\right\|_{H^{1}\left(K_{i}\right)} \lesssim \eta_{i}^{-\frac{1}{2}}\left|p_{i, K}\right|\left|K_{i}\right|^{\frac{1}{2}}=\left\|\eta^{-\frac{1}{2}} p_{i, h}\right\|_{L^{2}\left(K_{i}\right)} .
$$

- If $K \sim Q$, we cannot apply the previous construction of a suitable discrete velocity with local support. Hence, assume that $K$ is in a patch $P_{1}=\left\{K, K^{\prime}\right\}$, sharing an edge $E$ with an element $K^{\prime} \sim T$; let $E^{\prime}=E_{K^{\prime}}$. In this case, the idea is to build a discrete velocity having support localized on the patch $K \cup K^{\prime}$. Specifically, we set

$$
\widetilde{\mathbf{v}}_{i, P_{1}, h}=\left(m_{E} \boldsymbol{\phi}_{E}+m_{E^{\prime}} \phi_{E^{\prime}}\right)_{\mid \Omega_{i}} \in \mathbf{V}_{i, h} .
$$

Again, note that this function is localized on the patch as all degrees of freedom associated with edges on $\Gamma_{i, h}$ or shared with other elements of $\mathcal{G}_{h}$ are inactive. Choosing $m_{E}=-\eta_{i}^{-1} \frac{|K|}{|E|} p_{i, K}$ yields $\left(\nabla \cdot \widetilde{\mathbf{v}}_{i, P_{1}, h}\right)_{\mid K}=$ $-\eta_{i}^{-1} p_{i, K}$, irrespective of $m_{E^{\prime}} \operatorname{since} \operatorname{supp}\left(\phi_{E^{\prime}}\right)=K^{\prime}$. Then, we have $\left(\nabla \cdot \widetilde{\mathbf{v}}_{i, P_{1}, h}\right)_{\mid K^{\prime}}=m_{E^{\prime}} \frac{\left|E^{\prime}\right|}{\left|K^{\prime}\right|}-m_{E} \frac{|E|}{\left|K^{\prime}\right|}$, so that for $m_{E^{\prime}}=\frac{|E|}{\left|E^{\prime}\right|} m_{E}-\eta_{i}^{-1} \frac{\left|K^{\prime}\right|}{\left|E^{\prime}\right|} p_{i, K^{\prime}}=-\eta_{i}^{-1}\left[\frac{|K|}{\left|E^{\prime}\right|} p_{i, K}+\frac{\left|K^{\prime}\right|}{\left|E^{\prime}\right|} p_{i, K^{\prime}}\right]$ we get $\left(\nabla \cdot \widetilde{\mathbf{v}}_{i, P_{1}, h}\right)_{\mid K^{\prime}}=-\eta_{i}^{-1} p_{i, K^{\prime}}$. With this construction, we have $b\left(p_{h}, \widetilde{\mathbf{v}}_{i, P_{1}, h}\right)=\left\|\eta^{-\frac{1}{2}} p_{h}\right\|_{L^{2}\left(\Delta_{i}\right)}^{2}, \Delta_{i}=K_{i} \cup K_{i}^{\prime}$. On the other hand, proceeding as in the previous case, observing that $\operatorname{supp}\left(\phi_{E}\right) \cap \Omega_{i}=\Delta_{i}, \operatorname{supp}\left(\phi_{E^{\prime}}\right) \cap \Omega_{i}=K_{i}^{\prime}$, we have

$$
\begin{aligned}
\left\|\eta_{i}^{\frac{1}{2}} \widetilde{\mathbf{v}}_{i, P_{1}, h}\right\|_{H^{1}\left(\Delta_{i}\right)} & \lesssim \eta_{i}^{\frac{1}{2}} h^{-1}\left(\left|\Delta_{i}\right|^{\frac{1}{2}}\left|m_{E}\right|+\left|K_{i}^{\prime}\right|^{\frac{1}{2}}\left|m_{E^{\prime}}\right|\right) \lesssim \eta_{i}^{-\frac{1}{2}}\left(\left|\Delta_{i}\right|^{\frac{1}{2}}\left|p_{i, K}\right|+\left|K_{i}^{\prime}\right|^{\frac{1}{2}}\left|p_{i, K^{\prime}}\right|\right) \\
& \lesssim \eta_{i}^{-\frac{1}{2}}\left(\left|K_{i}\right|^{\frac{1}{2}}\left|p_{i, K}\right|+\left|K_{i}^{\prime}\right|^{\frac{1}{2}}\left|p_{i, K^{\prime}}\right|\right) \lesssim\left\|\eta^{-\frac{1}{2}} p_{h}\right\|_{L^{2}\left(\Delta_{i}\right)},
\end{aligned}
$$

where we used $\left|\Delta_{i}\right|=\left|K_{i}\right|+\left|K_{i}^{\prime}\right| \lesssim\left|K_{i}\right|$ thanks to (4.7). Note how we "tune" the velocity on $K_{i}$ by first acting on $m_{E}$. Since $E$ is shared with $K^{\prime}, m_{E}$ affects the velocity also in $K^{\prime}$; but we can still use $m_{E^{\prime}}$ as a corrector on $K^{\prime}$, with no consequences on neighboring elements of $\mathcal{G}_{h}$ since $K^{\prime} \sim T$ (i.e. $\phi_{E^{\prime}}$ has local support in $\left.K^{\prime}\right)$.

- If $K$ is in a patch $P_{n}=\left\{K^{1}, \ldots, K^{n} \sim Q ; K^{\prime} \sim T\right\}$, we proceed as in the previous case. Let $E^{\prime}=E_{K^{\prime}}$, and let $E^{j}$ be the edge shared by $K^{j}$ and $K^{j+1}$ if $j=1, \ldots, n-1$, and by $K^{n}$ and $K^{\prime}$ if $j=n$. Again, the idea is to build a discrete velocity having support localized on the patch, defining

$$
\widetilde{\mathbf{v}}_{i, P_{n}, h}=\left(m_{E^{1}} \boldsymbol{\phi}_{E^{1}}+m_{E^{2}} \boldsymbol{\phi}_{E^{2}}+\ldots+m_{E^{n}} \phi_{E^{n}}+m_{E^{\prime}} \boldsymbol{\phi}_{E^{\prime}}\right)_{\mid \Omega_{i}} \in \mathbf{V}_{i, h},
$$

where $\phi_{E^{j}} \cdot \mathbf{n}_{K^{j}}=1$ on $E^{j}, \phi_{E^{j}} \cdot \mathbf{n}_{K^{j}}=0$ on $\partial K^{j} \backslash E^{j}$. Again, we proceed by choosing:

$$
\begin{aligned}
& m_{E^{1}}=-\eta_{i}^{-1} \frac{\left|K^{1}\right|}{\left|E^{1}\right|} p_{i, K^{1}} \quad \text { s.t.: }\left(\nabla \cdot \widetilde{\mathbf{v}}_{i, P_{n}, h}\right)_{\mid K^{1}}=-\eta_{i}^{-1} p_{i, K^{1}} ; \\
& m_{E^{2}}=\frac{\left|E^{1}\right|}{\left|E^{2}\right|} m_{E^{1}}-\eta_{i}^{-1} \frac{\left|K^{2}\right|}{\left|E^{2}\right|} p_{i, K^{2}} \text { s.t.: }\left(\nabla \cdot \widetilde{\mathbf{v}}_{i, P_{n}, h}\right)_{\mid K^{2}}=-\eta_{i}^{-1} p_{i, K^{2}} ; \\
& \vdots \\
& m_{E^{\prime}}=\frac{\left|E^{n}\right|}{\left|E^{\prime}\right|} m_{E^{n}}-\eta_{i}^{-1} \frac{\left|K^{\prime}\right|}{\left|E^{\prime}\right|} p_{i, K^{\prime}} \text { s.t.: }\left(\nabla \cdot \widetilde{\mathbf{v}}_{i, P_{n}, h}\right)_{\mid K^{\prime}}=-\eta_{i}^{-1} p_{i, K^{\prime}} .
\end{aligned}
$$

We have $\left|m_{E^{j}}\right| \lesssim \eta^{-1} h\left(\left|p_{i, K^{1}}\right|+\ldots+\left|p_{i, K^{j-1}}\right|\right),\left|m_{E^{\prime}}\right| \lesssim \eta^{-1} h\left(\left|p_{i, K^{1}}\right|+\ldots+\left|p_{i, K^{n}}\right|\right)$. Let $\Delta_{i}^{j}=\bigcup_{k=j, \ldots, n} K_{i}^{j} \cup$ $K_{i}^{\prime}$, and denote by $\Delta_{i}=\Delta_{i}^{1}$ the whole patch; we have

$$
\begin{aligned}
\left\|\eta_{i}^{\frac{1}{2}} \widetilde{\mathbf{v}}_{i, P_{n}, h}\right\|_{H^{1}\left(\Delta_{i}\right)} \lesssim & \eta_{i}^{-\frac{1}{2}}\left(\left|\Delta_{i}^{1}\right|^{\frac{1}{2}}\left|p_{i, K^{1}}\right|+\left|\Delta_{i}^{2}\right|^{\frac{1}{2}}\left|p_{i, K^{2}}\right|+\left|\Delta_{i}^{3}\right|^{\frac{1}{2}}\left|p_{i, K^{3}}\right|+\ldots\right. \\
& \left.+\left|K_{i}^{n} \cup K_{i}^{\prime}\right|^{\frac{1}{2}}\left|p_{i, K^{n}}\right|+\left|K_{i}^{\prime}\right|^{\frac{1}{2}}\left|p_{i, K^{\prime}}\right|\right) \\
\lesssim & \eta_{i}^{-\frac{1}{2}}\left(\left|K_{i}^{1}\right|^{\frac{1}{2}}\left|p_{i, K^{1}}\right|+\ldots+\left|K_{i}^{n}\right|^{\frac{1}{2}}\left|p_{i, K^{n}}\right|+\left|K_{i}^{\prime}\right|^{\frac{1}{2}}\left|p_{i, K^{\prime}}\right|\right) \lesssim\left\|\eta^{-\frac{1}{2}} p_{h}\right\|_{L^{2}\left(\Delta_{i}\right)},
\end{aligned}
$$

where, again, estimates (4.7) have been used (note that $n$ is bounded by a moderate constant due to shape regularity). Note that the position of $K^{\prime}$ in the patch is not affecting the validity of our estimates. 
Now consider $\mathbf{v}_{p, h}=\sum_{i=1,2} \overline{\mathbf{v}}_{i, h}+\widetilde{\mathbf{v}}_{i, h} \in \mathbf{V}_{h}$, where $\widetilde{\mathbf{v}}_{i, h}=\sum_{P_{j}} \widetilde{\mathbf{v}}_{i, P_{j}, h}$, including all the patches $P_{j}$ covering $\mathcal{G}_{h}$. We have, by construction,

$$
b\left(p_{h}, \mathbf{v}_{p, h}\right)=\left\|\eta^{-\frac{1}{2}} p_{h}\right\|_{L^{2}(\Omega)}=\left\|p_{h}\right\|_{Q_{h}}, \quad \sum_{i}\left\|\eta^{\frac{1}{2}} \mathbf{v}_{i, p, h}\right\|_{H^{1}\left(\Omega_{i}\right)} \lesssim\left\|p_{h}\right\|_{Q_{h}}
$$

Finally, let us estimate the terms in $\left\|\mathbf{v}_{p, h}\right\|_{\mathbf{V}_{h}}$ that are not immediately controlled by $\sum_{i}\left\|\eta \eta_{u}^{\frac{1}{2}} \mathbf{v}_{i, p, h}\right\|_{H^{1}\left(\Omega_{i}\right)}$. As concerns the boundary terms, $\left\|h^{-\frac{1}{2}} \mathbf{v}_{p, h} \cdot \mathbf{n}\right\|_{L^{2}\left(\Gamma_{D}\right)}^{2}=0$. To conclude, we need to estimate the interface terms $\left\|\eta_{\Gamma}^{\frac{1}{2}} \mathbf{v}_{i, p, h} \cdot \mathbf{n}_{\Gamma}\right\|_{L^{2}(\Gamma)}, i=1,2$, in order to find an upper bound for $\left\|\eta_{\Gamma}^{\frac{1}{2}}\left\{\mathbf{v}_{p, h} \cdot \mathbf{n}_{\Gamma}\right\}\right\|_{L^{2}(\Gamma)}^{2}$ and $\left\|\eta_{\Gamma}^{\frac{1}{2}} \llbracket \mathbf{v}_{p, h} \cdot \mathbf{n}_{\Gamma} \rrbracket\right\|_{L^{2}(\Gamma)}^{2}$. To this end, we first observe that $\left\|\mathbf{v}_{i, p, h} \cdot \mathbf{n}_{\Gamma}\right\|_{L^{2}(\Gamma)}^{2}=\sum_{K \in \mathcal{G}_{h}} \int_{\Gamma_{K}}\left(\widetilde{\mathbf{v}}_{i, h} \cdot \mathbf{n}_{\Gamma}\right)^{2}$. For each $K \in \mathcal{G}_{h}$, the function $\widetilde{\mathbf{v}}_{i, h}$ is vanishing at some point of $\Gamma_{i, h}$ (at a vertex, if $K \sim T$; at some point of the only edge lying on $\Gamma_{i, h}$, otherwise); as a consequence, we have $\left\|\widetilde{\mathbf{v}}_{i, h}\right\|_{L^{2}\left(\Gamma_{K}\right)} \lesssim h\left|\mathbf{v}_{i, p, h}\right|_{H^{1}\left(K_{i}\right)}$ yielding $\left\|\eta_{\Gamma}^{\frac{1}{2}} \mathbf{v}_{i, p, h}\right\|_{L^{2}(\Gamma)} \lesssim \eta_{\Gamma}^{\frac{1}{2}} h\left\|\eta_{i}^{-1} p_{i, h}\right\|_{L^{2}\left(\Omega_{i}\right)}=$ $h\left(\frac{\eta_{\Gamma}}{\eta_{i}}\right)^{\frac{1}{2}}\left\|p_{h}\right\|_{Q_{h}}$. Collecting all the estimates, we have

$$
\left\|\widetilde{\mathbf{v}}_{h}\right\|_{\mathbf{V}_{h}} \lesssim M_{\Gamma}\left\|p_{h}\right\|_{Q_{h}}, \quad M_{\Gamma}=\max \left\{1, h\left(\frac{\eta_{\Gamma}}{\eta_{\min }}\right)^{1 / 2}\right\} .
$$

In other words, the fact that $\mathbf{v}_{i, p, h}$ is zero near $\Gamma$ (but not exactly zero on $\Gamma$ ) provides us with a mild dependence of the stability estimate on the coefficients, which is asymptotically robust for $h \rightarrow 0$.

Remark 4.9. The procedure of dividing $\mathcal{G}_{h}$ in patches and constructing velocities with local support on the patches is rather technical, but also necessary. It seems to us that simpler approaches do not allow to obtain the same result. As an example, let us consider $p_{i, h} \in Q_{i, h}, \operatorname{supp}\left(p_{i, h}\right) \subset \mathcal{G}_{h} \cap \Omega_{i}$. Let $p_{i, h}^{*}$ a piecewise constant function such that $p_{i, h \mid K}^{*}=p_{i, h \mid K_{i}}$ for all $K \in \mathcal{G}$. Let $\mathbf{v}_{i, p, h}^{*}$ be the classical Fortin's interpolant associated to $p_{i, h}^{*}$; we can set $\mathbf{v}_{i, p, h}=\left(\mathbf{v}_{i, p, h}^{*}\right)_{\mid \Omega_{i}} \in \mathbf{V}_{i, h}$, and since $\nabla \cdot \mathbf{v}_{i, p, h}^{*}=-p_{i, h}^{*}$ on each $K \in \mathcal{G}, b\left(p_{i, h}, \mathbf{v}_{i, p, h}\right)=$ $\left\|p_{i, h}\right\|_{L^{2}\left(\Omega_{i}\right)}^{2}$. But we would only have the estimate $\left\|\mathbf{v}_{i, p, h}\right\|_{1, \Omega_{i}}^{2} \lesssim \sum_{K \in \mathcal{G}_{h}}\left\|p_{i, h}^{*}\right\|_{L^{2}(K)}^{2}$, that we cannot control by $\sum_{K \in \mathcal{G}_{h}}\left\|p_{i, h}^{*}\right\|_{L^{2}\left(K_{i}\right)}^{2}=\left\|p_{i, h}\right\|_{L^{2}\left(\Omega_{i}\right)}^{2}\left(\left|K_{i}\right|\right.$ can be arbitrarily small w.r.t. $\left.|K|\right)$.

The inf-sup condition implies the following stability result.

Theorem 4.10 (stability). Let $\left(\mathbf{u}_{h}, p_{h}\right) \in \mathbf{W}_{h}$, and let $M_{\Gamma}$ be the constant of Lemma 4.8. Then,

$$
\left\|\left(\mathbf{u}_{h}, p_{h}\right)\right\|_{\mathbf{W}_{h}} \lesssim M_{\Gamma} \sup _{\left(\mathbf{v}_{h}, q_{h}\right) \in \mathbf{W}_{h}} \frac{\mathcal{C}\left(\left(\mathbf{u}_{h}, p_{h}\right),\left(\mathbf{v}_{h}, q_{h}\right)\right)}{\left\|\left(\mathbf{v}_{h}, q_{h}\right)\right\|_{\mathbf{W}_{h}}} .
$$

Proof. Let $\mathbf{v}_{p, h}$ be the function associated to $p_{h}$ as in Lemma 4.8, and let

$$
\left(\mathbf{v}_{h}, q_{h}\right)=\left(\mathbf{u}_{h}+\delta_{1} \mathbf{v}_{p, h}, p_{h}+\delta_{2} \eta \nabla \cdot \mathbf{u}_{h}\right), \quad \delta_{1}, \delta_{2}>0 .
$$

We shall first show that $\mathcal{C}\left(\left(\mathbf{u}_{h}, p_{h}\right),\left(\mathbf{v}_{h}, q_{h}\right)\right) \gtrsim\left\|\left(\mathbf{u}_{h}, p_{h}\right)\right\|_{\mathbf{W}_{h}}^{2}$; next, we will check that $\left\|\left(\mathbf{v}_{h}, q_{h}\right)\right\|_{\mathbf{W}_{h}}^{2} \lesssim$ $M_{\Gamma}\left\|\left(\mathbf{u}_{h}, p_{h}\right)\right\|_{\mathbf{W}_{h}}^{2}$. For the first part, we exploit the bilinearity of $\mathcal{C}$ to obtain,

$$
\mathcal{C}\left(\left(\mathbf{u}_{h}, p_{h}\right),\left(\mathbf{v}_{h}, q_{h}\right)\right)=\mathcal{C}\left(\left(\mathbf{u}_{h}, p_{h}\right),\left(\mathbf{u}_{h}, p_{h}\right)\right) \delta_{1} \mathcal{C}\left(\left(\mathbf{u}_{h}, p_{h}\right),\left(\mathbf{v}_{p, h}, 0\right)\right)+\delta_{2} \mathcal{C}\left(\left(\mathbf{u}_{h}, p_{h}\right),\left(0, \eta \nabla \cdot \mathbf{u}_{h}\right)\right) .
$$

Thanks to Lemma 4.4 we have

$$
\mathcal{C}\left(\left(\mathbf{u}_{h}, p_{h}\right),\left(\mathbf{u}_{h}, p_{h}\right)\right) \geq \|\left.\left|\mathbf{u}_{h}\right|\right|^{2} .
$$

Let us consider the second term of (4.8). Thanks to Lemmas 4.3, 4.8, for all $\epsilon_{1}>0$ we get

$$
a\left(\mathbf{u}_{h}, \mathbf{v}_{p, h}\right) \lesssim||\left|\mathbf{u}_{h}\right|\left\|||\left|\mathbf{v}_{p, h}\right|\right\|\left|\leq \frac{1}{\epsilon_{1}}\right||| \mathbf{u}_{h}\left|\left\|^{2}+\epsilon_{1}\left|\left\|\mathbf{v}_{p, h}\left|\left\|^{2} \lesssim \frac{1}{\epsilon_{1}}\right\|\right| \mathbf{u}_{h}||^{2}+\epsilon_{1}\right\| \eta^{-1} p_{h} \|_{L^{2}(\Omega)}^{2},\right.\right.\right.
$$


so that

$$
\begin{aligned}
\mathcal{C}\left(\left(\mathbf{u}_{h}, p_{h}\right),\left(\mathbf{v}_{p, h}, 0\right)\right) & =a\left(\mathbf{u}_{h}, \mathbf{v}_{p, h}\right)+b\left(p_{h}, \mathbf{v}_{p, h}\right) \\
& \geq b\left(p_{h}, \mathbf{v}_{p, h}\right)-C_{1} \epsilon_{1}\left\|p_{h}\right\|_{Q_{h}}^{2}-\frac{C_{1}}{\epsilon_{1}}\left\|\mathbf{u}_{h}\right\| \|^{2} .
\end{aligned}
$$

Then, thanks again to Lemma 4.8 we have

$$
\mathcal{C}\left(\left(\mathbf{u}_{h}, p_{h}\right),\left(\mathbf{v}_{p, h}, 0\right)\right) \gtrsim\left(1-C_{1} \epsilon_{1}\right)\left\|p_{h}\right\|_{Q_{h}}^{2}-\frac{C_{1}}{\epsilon_{1}}\left\|\left|\mathbf{u}_{h} \|\right|^{2} .\right.
$$

Now consider the third term in equation (4.8). Thanks to standard inverse inequalities, we can estimate

$$
\begin{aligned}
-\int_{E}\left(\eta \nabla \cdot \mathbf{u}_{h}\right)\left(\mathbf{u}_{h} \cdot \mathbf{n}\right) & \leq\left\|h^{\frac{1}{2}} \eta^{\frac{1}{2}}\left(\nabla \cdot \mathbf{u}_{h}\right)\right\|_{L^{2}(E)}\left\|h^{\frac{1}{2}} \eta^{-\frac{1}{2}} \mathbf{u}_{h} \cdot \mathbf{n}\right\|_{L^{2}(E)} \\
& \lesssim \epsilon_{2}\left\|\eta^{\frac{1}{2}} \nabla \cdot \mathbf{u}_{h}\right\|_{L^{2}(K)}^{2}+\frac{1}{\epsilon_{2}}\left\|h^{-\frac{1}{2}} \eta^{\frac{1}{2}} \mathbf{u}_{h} \cdot \mathbf{n}\right\|_{L^{2}(E)}^{2}
\end{aligned}
$$

for all edges $E \subset \Gamma_{D} \cap \partial K, K \in \mathcal{T}_{h}$, and any $\epsilon_{2}>0$. As a consequence,

$$
\begin{aligned}
\mathcal{C}\left(\left(\mathbf{u}_{h}, p_{h}\right),\left(0, \eta \nabla \cdot \mathbf{u}_{h}\right)\right) & =\left\|\eta^{\frac{1}{2}} \nabla \cdot \mathbf{u}_{h}\right\|_{L^{2}(\Omega)}^{2}-\int_{\Gamma_{D}}\left(\eta \nabla \cdot \mathbf{u}_{h}\right)\left(\mathbf{u}_{h} \cdot \mathbf{n}\right) \\
& \geq\left(1-C_{2} \epsilon_{2}\right)\left\|\eta^{\frac{1}{2}} \nabla \cdot \mathbf{u}_{h}\right\|_{0, \Omega}^{2}-\frac{C_{2}}{\epsilon_{2}}\left\|\eta^{\frac{1}{2}} \mathbf{u}_{h} \cdot \mathbf{n}\right\|_{h,+\frac{1}{2}, \Gamma_{D}}^{2}
\end{aligned}
$$

Collecting the previous results, and observing that $\left\|\eta^{\frac{1}{2}} \mathbf{u}_{h} \cdot \mathbf{n}\right\|_{h,+\frac{1}{2}, \Gamma_{D}}^{2} \lesssim\|\| \mathbf{u}_{h}\|\|$, we get

$$
\begin{aligned}
\mathcal{C}\left(\left(\mathbf{u}_{h}, p_{h}\right),\left(\mathbf{v}_{h}, q_{h}\right)\right) \gtrsim & \left(1-C_{1} \frac{\delta_{1}}{\epsilon_{1}}-C_{2} \frac{\delta_{2}}{\epsilon_{2}}\right)\|\| \mathbf{u}_{h}\|\|^{2}+\delta_{1}\left(1-C_{1} \epsilon_{1}\right)\left\|p_{h}\right\|_{Q_{h}}^{2} \\
& +\delta_{2}\left(1-C_{2} \epsilon_{2}\right)\left\|\eta^{\frac{1}{2}} \nabla \cdot \mathbf{u}_{h}\right\|_{L^{2}(\Omega)}^{2} .
\end{aligned}
$$

Now, let us choose sufficiently small parameters $\epsilon_{i}$ and $\delta_{i}$ such that all the norms in (4.10) are multiplied by positive coefficients. For instance, take $\epsilon_{i}=1 /\left(2 C_{i}\right)$ and $\delta_{i}=1 /\left(8 C_{i}^{2}\right)$. From (4.10) we get

$$
\mathcal{C}\left(\left(\mathbf{u}_{h}, p_{h}\right),\left(\mathbf{v}_{h}, q_{h}\right)\right) \gtrsim\left|\left\|\mathbf{u}_{h} \mid\right\|^{2}+\left\|\eta^{-\frac{1}{2}} p_{h}\right\|_{L^{2}(\Omega)}^{2}+\left\|\eta^{\frac{1}{2}} \nabla \cdot \mathbf{u}_{h}\right\|_{L^{2}(\Omega)}^{2}=\left\|\left(\mathbf{u}_{h}, p_{h}\right)\right\|_{\mathbf{W}_{h}}^{2} .\right.
$$

For the second part of the proof, since $\delta_{i} \lesssim 1$, and $\left\|\left(0, \eta \nabla \cdot \mathbf{u}_{h}\right)\right\|_{\mathbf{W}_{h}} \lesssim\left\|\left(\mathbf{u}_{h}, p_{h}\right)\right\|_{\mathbf{w}_{h}}$, we have $\left\|\left(\mathbf{v}_{h}, q_{h}\right)\right\|_{\mathbf{W}_{h}} \lesssim$ $\left\|\left(\mathbf{u}_{h}, p_{h}\right)\right\|_{\mathbf{w}_{h}}+\delta_{1}\left\|\left(\mathbf{v}_{p, h}, 0\right)\right\|_{\mathbf{w}_{h}}$. Moreover, using Lemma 4.8,

$$
\delta_{1}\left\|\left(\mathbf{v}_{p, h}, 0\right)\right\|_{\mathbf{W}_{h}} \lesssim M_{\Gamma}\left\|p_{h}\right\|_{Q_{h}} \lesssim M_{\Gamma}\left\|\left(\mathbf{u}_{h}, p_{h}\right)\right\|_{\mathbf{w}_{h}} .
$$

Based on this stability result, we can state in a standard way the convergence of our numerical scheme.

Theorem 4.11 (convergence). Let $(\mathbf{u}, p) \in \mathbf{W}$ be the solution of problem (2.1). There exist a unique solution $\left(\mathbf{u}_{h}, p_{h}\right) \in \mathbf{W}_{h}$ of the discrete problem (3.4), and

$$
\left\|\left(\mathbf{u}_{h}-\mathbf{u}, p_{h}-p\right)\right\|_{\mathbf{W}_{h}} \lesssim M_{\Gamma} \inf _{\left(\mathbf{v}_{h}, q_{h}\right) \in \mathbf{W}_{h}}\left\|\left(\mathbf{v}_{h}-\mathbf{u}, q_{h}-p\right)\right\|_{\mathbf{w}_{h}} .
$$

In particular, if $\mathbf{u}_{i} \in \boldsymbol{H}^{2}\left(\Omega_{i}\right), p_{i} \in H^{1}\left(\Omega_{i}\right)$, we have

$$
\left\|\left(\mathbf{u}_{h}-\mathbf{u}, p_{h}-p\right)\right\|_{\mathbf{w}_{h}} \lesssim M_{\Gamma}\left[h \sum_{i=1,2}\left(\left|\eta^{\frac{1}{2}} \nabla \cdot \mathbf{u}_{i}\right|_{H^{1}\left(\Omega_{i}\right)}+\left|\eta^{-\frac{1}{2}} p_{i}\right|_{H^{1}\left(\Omega_{i}\right)}\right)\right] .
$$


Proof. By Theorem 4.10, the matrix associated with $\mathcal{C}$ evaluated on $\mathbf{W}_{h} \times \mathbf{W}_{h}$ is non-singular (precisely, its smallest singular value is positive). Hence, there exists a unique discrete solution $\left(\mathbf{u}_{h}, p_{h}\right)$ of (3.4). Moreover, by using stability, consistency and boundedness (Thm. 4.10 and Lems. 4.1, 4.3) we get, for any $\left(\mathbf{v}_{h}, q_{h}\right) \in \mathbf{W}_{h}$,

$$
\begin{aligned}
\left\|\left(\mathbf{u}_{h}-\mathbf{u}, p_{h}-p\right)\right\| \mathbf{w}_{h} & \leq\left\|\left(\mathbf{v}_{h}-\mathbf{u}, q_{h}-p\right)\right\| \mathbf{w}_{h}+\left\|\left(\mathbf{v}_{h}-\mathbf{u}_{h}, q_{h}-p_{h}\right)\right\| \mathbf{w}_{h} \\
& \lesssim\left\|\left(\mathbf{v}_{h}-\mathbf{u}, q_{h}-p\right)\right\|_{\mathbf{w}_{h}}+M_{\Gamma} \sup _{\left(\mathbf{v}_{h}^{\prime}, q_{h}^{\prime}\right) \in \mathbf{W}_{h}} \frac{\mathcal{C}\left(\left(\mathbf{v}_{h}-\mathbf{u}_{h}, q_{h}-p_{h}\right),\left(\mathbf{v}_{h}^{\prime}, q_{h}^{\prime}\right)\right)}{\left\|\left(\mathbf{v}_{h}^{\prime}, q_{h}^{\prime}\right)\right\|_{\mathbf{W}_{h}}} \\
& =\left\|\left(\mathbf{v}_{h}-\mathbf{u}, q_{h}-p\right)\right\|_{\mathbf{w}_{h}}+M_{\Gamma} \sup _{\left(\mathbf{v}_{h}^{\prime}, q_{h}^{\prime}\right) \in \mathbf{W}_{h}} \frac{\mathcal{C}\left(\left(\mathbf{v}_{h}-\mathbf{u}, q_{h}-p\right),\left(\mathbf{v}_{h}^{\prime}, q_{h}^{\prime}\right)\right)}{\left\|\left(\mathbf{v}_{h}^{\prime}, q_{h}^{\prime}\right)\right\|_{\mathbf{w}_{h}}} \\
& \lesssim M_{\Gamma}\left\|\left(\mathbf{v}_{h}-\mathbf{u}, q_{h}-p\right)\right\|_{\mathbf{W}_{h},}
\end{aligned}
$$

so that (4.12) follows. Equation (4.13) is then obtained by taking $\mathbf{v}_{h}=I_{h}^{*} \mathbf{u}, q_{h}=C_{h}^{*} p$, where $I_{h}^{*}: H^{2}\left(\Omega_{1}\right) \times$ $H^{2}\left(\Omega_{2}\right) \rightarrow \mathbf{V}_{h}$ is an extended Raviart-Thomas intepolant and $C_{h}^{*}: H^{1}\left(\Omega_{1}\right) \times H^{1}\left(\Omega_{2}\right) \rightarrow Q_{h}$ is an extended piecewise constant interpolant. Based on the results obtained in [6], these interpolant can be easily constructed. In particular, given $\mathbf{u}=\left(\mathbf{u}_{1}, \mathbf{u}_{2}\right) \in H^{2}\left(\Omega_{1}\right) \times H^{2}\left(\Omega_{2}\right)$, let $\mathbf{u}_{i}^{*}=E_{i}^{*} \mathbf{u}_{i}$ where $E_{i}^{*}: H^{2}\left(\Omega_{i}\right) \rightarrow H^{2}(\Omega)$ is a continuous extension operator. Let $I_{i, h}$ be the standard $\mathbb{R} \mathbb{T}_{0}$ interpolant, and define $I_{h}^{*} \mathbf{u}=\left(I_{1, h} \mathbf{u}_{1}, I_{2, h} \mathbf{u}_{2}\right) \in \mathbf{V}_{h}$. The interpolant $C_{h}^{*}$ is defined similarly (and is the same of [6], Thm. 3). Proceeding as in [6], Theorem 3, estimates of the interpolation error for of the form $\left\|\mathbf{v}-I_{h}^{*} \mathbf{v}\right\|_{\mathbf{v}_{h}} \lesssim h \sum_{i}\left|\eta_{i}^{\frac{1}{2}} \mathbf{v}_{i}\right|_{H^{2}\left(\Omega_{i}\right)}$ and $\left\|q-C_{h}^{*} q\right\|_{Q_{h}} \lesssim h \sum_{i}\left|\eta_{i}^{-\frac{1}{2}} q_{i}\right|_{H^{1}\left(\Omega_{i}\right)}$ are obtained.

Let us comment on the robustness of the stability estimate in Lemma 4.10, i.e., on the dependence of $M_{\Gamma}$ on the coefficients. Since $M_{\Gamma}=\max \left\{1, h \sqrt{\eta_{\Gamma} / \eta_{\min }}\right\}$, the estimate is asymptotically robust with respect to the coefficients $\eta_{\Gamma}, \eta$, as $h \rightarrow 0$ (in which case $M_{\Gamma} \rightarrow 1$ ), the only issue being having a large $\eta_{\Gamma}$ compared to $\eta_{\min }$ when using "coarse" grids. We recall that this behavior is due to the fact that we are not allowed to choose function $\mathbf{v}_{p, h}$ of Lemma 4.8 such that $\mathbf{v}_{i, h} \cdot \mathbf{n}_{\Gamma}=0$ on $\Gamma$ (the proof only ensured that the normal components are $O(h))$.

We also recall that the norms are depending on parameters: for instance, $\eta$ appears in the norms $\|\cdot\|_{\mathbf{V}_{h}}$ and $\|\cdot\|_{Q_{h}}$, weighting the velocity and the pressure with antagonistic actions. Velocities are weighted by $\eta^{\frac{1}{2}}$, pressures by $\eta^{-\frac{1}{2}}$. This means that in highly permeable regions $(\eta \ll 1)$ our norms mostly control the pressure, whilst in almost impervious regions $(\eta \gg 1)$ they mostly control the velocity.

Finally, our assumption that $\eta_{i}$ is constant on each subdomain $\Omega_{i}$ is obviously very restrictive; in the more general case $\eta \in Q_{h}, M_{\Gamma}$ would also depend on the ratios $\eta_{i, \max } / \eta_{i, \min }$ (see [6]).

\subsection{A natural preconditioner}

Let us consider the algebraic properties of the discrete problem (3.4). As in [11], we expect the robustness of the stability estimate to be an advantage at the algebraic level. With little abuse of notation, $\mathbf{u}_{h} \in \mathbf{V}_{h}$ and $p_{h} \in Q_{h}$ will also denote the vectors $\mathbf{u}_{h} \in \mathbb{R}^{\operatorname{dim}\left(\mathbf{V}_{h}\right)}$ and $p_{h} \in \mathbb{R}^{\operatorname{dim}\left(Q_{h}\right)}$ associated to the respective finite element bases of the spaces $\mathbf{V}_{h}, Q_{h}$, and so for $\mathbf{v}_{h}, q_{h}$, etc. According to this notation, the linear system corresponding to problem (3.4) reads

$$
C\left[\begin{array}{l}
\mathbf{u}_{h} \\
p_{h}
\end{array}\right]=\left[\begin{array}{cc}
A & B^{T} \\
-B & 0
\end{array}\right]\left[\begin{array}{l}
\mathbf{u}_{h} \\
p_{h}
\end{array}\right]=\left[\begin{array}{l}
\boldsymbol{f}_{v} \\
f_{q}
\end{array}\right]
$$

whose blocks are related to the bilinear forms by $a(u, v)=(v, A u)$ and $b(q, u)=(q, B u)$, where $(\cdot, \cdot)$ is the Euclidean scalar product.

Let us equip $\mathbb{R}^{\operatorname{dim}\left(\mathbf{V}_{h}\right)} \times \mathbb{R}^{\operatorname{dim}\left(Q_{h}\right)}$ with the norm

$$
\left.||\left|\mathbf{v}_{h}, q_{h}\right|\right|^{2}=\left(\mathbf{v}_{h}, H_{V} \mathbf{v}_{h}\right)_{2}+\left(p_{h}, H_{Q} p_{h}\right)_{2}
$$


being $H_{V}$ and $H_{Q}$ the symmetric, positive matrices inducing the natural norms on $\mathbf{V}_{h}$ and $Q_{h}$, i.e. $\left(\mathbf{v}_{h}, H_{V} \mathbf{v}_{h}\right)_{2}=\left\|\mathbf{v}_{h}\right\|_{\mathbf{V}_{h}}^{2},\left(q_{h}, H_{Q} q_{h}\right)_{2}=\left\|q_{h}\right\|_{Q_{h}}^{2}$. Consider the following block-diagonal, symmetric and positive definite matrix,

$$
P=\left[\begin{array}{cc}
H_{V} & 0 \\
0 & H_{Q}
\end{array}\right]
$$

With a simple algebraic manipulation (see also [11] for a similar analysis), the boundedness and stability properties 4.3, 4.10 lead to the following estimates: for all $\mathbf{u}_{h} \in \mathbf{V}_{h}, p_{h} \in Q_{h}$,

$$
M_{\Gamma}^{-1}\left\|\left(\mathbf{u}_{h}, p_{h}\right)\right\|_{2} \lesssim \sup _{\mathbf{v}_{h}, q_{h}} \frac{\left(\left(\mathbf{u}_{h}, p_{h}\right), P^{-\frac{1}{2}} C P^{-\frac{1}{2}}\left(\mathbf{v}_{h}, q_{h}\right)\right)_{2}}{\left\|\left(\mathbf{v}_{h}, q_{h}\right)\right\|_{2}} \lesssim\left\|\left(\mathbf{u}_{h}, p_{h}\right)\right\|_{2}
$$

Thanks to (4.16), we have that the singular values of the matrix $P^{-\frac{1}{2}} C P^{-\frac{1}{2}}$ are lower and upper bounded by positive constants $\underline{\sigma}, \bar{\sigma}$, such that $\underline{\sigma} \gtrsim M_{\Gamma}^{-1}$, and $\bar{\sigma}$ is independent of $h, \eta$ and $\eta_{\Gamma}$ (see also [8], Sect. II.3.1, and [11], Sect. 3 and Thm. 3.8; a general presentation of block-diagonal preconditioning of saddle point problems arising form stable mixed finite element approximation of Darcy's problem is also addressed in [17]). It follows that $\underline{\sigma} \leq\left|\lambda_{i}\left(P^{-1} C\right)\right| \leq \bar{\sigma}$, being $\lambda_{i}\left(P^{-1} C\right)$ the eigenvalues of the preconditioned matrix $P^{-1} C$. By changing the sign in the mass conservation equation, system (4.14) can be recast in a symmetric (indefinite) form. It follows that the $P$-preconditioned MinRes methods can be successfully employed at each time step: for a fixed tolerance, the number of iterations will be independent of $h$, and also of the physical parameters (at least asymptotically for $h \rightarrow 0$ ).

The preconditioner $P$ has a block-diagonal structure, each block being a symmetric positive definite matrix (SPD). Whilst the pressure block is a pressure mass matrix and is easily dealt with, the velocity block features a div-div term $\left(\eta \nabla \cdot \mathbf{u}_{h}, \nabla \cdot \mathbf{u}_{h}\right)_{\Omega}$, requiring suitable a sub-preconditioner to be used to efficiently solve systems associated to matrix $H_{V}$. A detailed analysis and an effective sub-preconditioning strategy was proposed by Arnold et al. in [4].

\subsection{Condition number of matrix $C$ and interface location}

\subsubsection{Full P-preconditioning}

In this section, we report numerical tests that support the fact that the extreme eigenvalues of matrix $P^{-1} C$ are independent of both $h$ and the position of the fracture $\Gamma$ with $P$ defined as in (4.15), i.e. we numerically verify (4.16). If $h$-optimality is an important feature of any preconditioner, $\Gamma$-optimality is even more important. Indeed, since each element $K \in \mathcal{G}_{h}$ is divided by $\Gamma$ into two sub-elements $K_{1}, K_{2}$, one could expect the conditioning of the matrix to degenerate as $\mu\left(\mathcal{T}_{h}, \Gamma\right)=\min _{K \in \mathcal{G} i=1,2} \min _{\left|K_{i}\right|} \mid \frac{|K|}{}$ tends to zero. This is a potentially critical situation, that must be carefully handled since we want the mesh to be irrespective of the position of $\Gamma$. We refer to [20] for a detailed analysis of this issue for the FEM approximation of contrast problems.

To study the spectrum of $C$ for different fracture configurations, we solved problem (2.1) on a square domain $\Omega=[0,1] \times[0,1]$, with (non-homogeneous) Dirichlet boundary conditions on $\partial \Omega$. We considered a regular triangulation, cut by a vertical embedded interface $\Gamma=\{\bar{x}\} \times[0,1]$, for different values of $\bar{x} \in(0,1)$ in order to obtain smaller and smaller values of $\mu\left(\mathcal{T}_{h}, \Gamma\right)$ by creating elements $K$ with small $\frac{\left|K_{i}\right|}{|K|}$ ratios.

That was obtained using structured meshes on $\Omega$ such that vertical midline $\Gamma_{M}=\{(x, y) \mid x=0.5\}$ was always covered by triangle edges, and taking $\bar{x} \rightarrow 0.5$, i.e. taking the interface $\Gamma$ very close to $\Gamma_{M}$.

Table 1 shows the maximum and minimum eigenvalues of the matrix $C$ for $\bar{x} \rightarrow 0.5$ (i.e., for $\mu\left(\mathcal{T}_{h}, \Gamma\right) \rightarrow 0$ ) for two grids of 200 and 800 triangles respectively. As $\mu\left(\mathcal{T}_{h}, \Gamma\right)$ tends to zero the eigenvalue of lowest module decreases, and the ratio between the maximum and minimum $\left|\lambda_{i}\right|$ is larger for the finest mesh. The maximum 
TABle 1. Extreme eigenvalues of $C$ and of $P^{-1} C$ for different positions $\bar{x}$ of the interface. Two regular meshes of $N_{h}=200$ (a) and $N_{h}=800$ (b) triangles are considered.

\begin{tabular}{c|c|c|c|c|c|c}
\hline & $\bar{x}$ & $\mu\left(\mathcal{T}_{h}, \Gamma\right)$ & $\max \left|\lambda_{i}(C)\right|$ & $\min \left|\lambda_{i}(C)\right|$ & $\max \left|\lambda_{i}\left(P^{-1} C\right)\right|$ & $\min \left|\lambda_{i}\left(P^{-1} C\right)\right|$ \\
\hline \multirow{5}{*}{ (a) } & 0.49 & $1.00 \mathrm{e}-2$ & 0.2805 & $0.1005 \mathrm{e}-3$ & 1 & 0.9895 \\
& 0.495 & $2.50 \mathrm{e}-3$ & 0.2805 & $0.0337 \mathrm{e}-3$ & 1 & 0.9897 \\
& 0.4975 & $6.25 \mathrm{e}-4$ & 0.2805 & $0.0128 \mathrm{e}-3$ & 1 & 0.9898 \\
& 0.499 & $1.00 \mathrm{e}-4$ & 0.2805 & $0.0045 \mathrm{e}-3$ & 1 & 0.9898 \\
& 0.4995 & $2.50 \mathrm{e}-5$ & 0.2805 & $0.0025 \mathrm{e}-3$ & 1 & 0.9898 \\
& 0.4999 & $1.00 \mathrm{e}-6$ & 0.2805 & $0.0001 \mathrm{e}-3$ & 1 & 0.9898 \\
& & & & & & 0.9895 \\
& 0.49 & $1.00 \mathrm{e}-2$ & 0.1411 & $0.1005 \mathrm{e}-4$ & 1 & 0.9897 \\
& 0.495 & $2.50 \mathrm{e}-3$ & 0.1411 & $0.0337 \mathrm{e}-4$ & 1 & 0.9898 \\
& 0.4975 & $6.25 \mathrm{e}-4$ & 0.1411 & $0.0128 \mathrm{e}-4$ & 1 & 0.9898 \\
& 0.499 & $1.00 \mathrm{e}-4$ & 0.1411 & $0.0045 \mathrm{e}-4$ & 1 & 0.9898 \\
& 0.4995 & $2.50 \mathrm{e}-5$ & 0.1411 & $0.0025 \mathrm{e}-4$ & 1 & 1 \\
\hline
\end{tabular}

and minimum eigenvalues of the preconditioned matrix instead are constant for any position of the interface and independent on the mesh size $h$, confirming the optimality of the preconditioner (4.15).

\subsubsection{Matrix equilibration}

As mentioned above the preconditioner $P$ is not easy to deal with due to the div-div term, therefore a simpler alternative would be desirable. We will provide experimental evidence of the fact that matrix equilibration (that is an instance of diagonal preconditioning) can make the conditioning of the discrete problem independent of the position of the interface. Of course, we loose the optimality with respect to $h$; nevertheless, the fact that the simple equilibration procedure (implemented by automatic routines in most linear algebra packages) can cure the potentially severe ill-conditioning due to small ratios $\left|K_{i}\right| /|K|$ is obviously very important. Here, we consider the following equilibration method. Consider the block diagonal matrix

$$
\tilde{P}=\left[\begin{array}{cc}
A & 0 \\
0 & H_{Q}
\end{array}\right]
$$

and denote by $P_{L}$ its lumped diagonal matrix, such that $\left(P_{L}\right)_{i i}=\sum_{j}(\tilde{P})_{i j}$. This diagonal preconditioner performs an effective scaling of the matrix: as shown in Figure 4 (left) the eigenvalues of $P_{L}^{-1} C$ remain constant as $\mu\left(\mathcal{T}_{h}, \Gamma\right)$ tends to zero and depend mildly on the mesh size. Of course, the equilibration algorithm is not unique and many other techniques could be applied as well, including automatic equilibration routines.

Finally, we point out that the limit case in which $\mu\left(\mathcal{T}_{h}, \Gamma\right)=0$ can be handled in practice eliminating the extended DOFs associated to "small" sub-elements [19].

\section{The COUPLED PROBlem: AN ITERATIVE METHOD}

\subsection{Fracture flow}

The weak formulation and finite element approximation of the fracture flow problem (2.5) is obtained following the same method employed for the bulk flow problem (2.1). Moreover, we now consider standard (i.e. not extended) finite element spaces $\hat{\mathbf{V}}_{h}=\left\{\hat{\mathbf{v}}_{h} \in \boldsymbol{H}_{\text {div }}(\Gamma): \hat{\mathbf{v}}_{\left.h\right|_{K}} \in \mathbb{R} \mathbb{T}_{0}(K) \forall K \in \hat{\mathcal{T}}_{h}\right\}, \hat{Q}_{h}=\left\{\hat{q}_{h} \in L^{2}(\Gamma):\right.$ $\left.\hat{q}_{\left.h\right|_{K}} \in \mathbb{P}_{0}(K) \forall K \in \hat{\mathcal{T}}_{h}\right\}, \hat{\mathbf{W}}_{h}=\hat{\mathbf{V}}_{h} \times \hat{Q}_{h}$. Let us introduce the following bilinear forms related to the fracture 


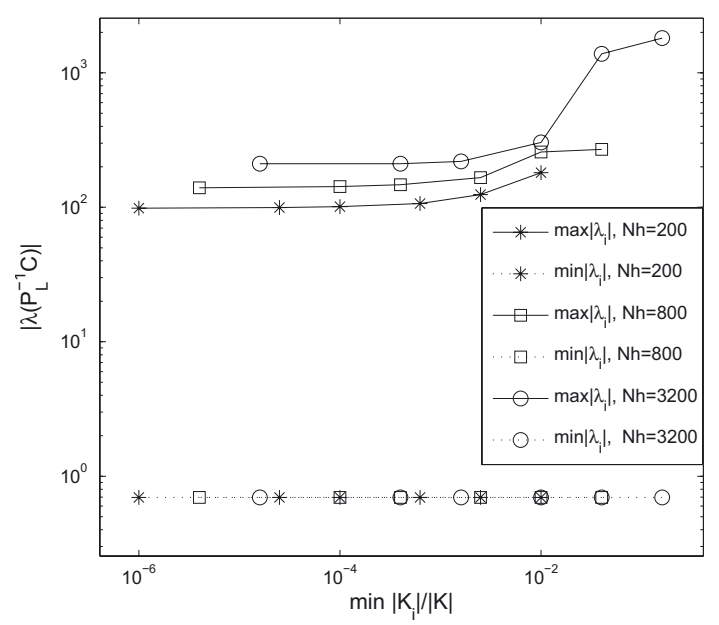

Figure 4. Extreme eigenvalues of $C$ preconditioned by the lumped preconditioner $P_{L}$ for different positions of the interface. Three different meshes of $N_{h}=200,800,3200$ triangles are considered.

flow,

$$
\begin{aligned}
& \hat{a}\left(\mathbf{u}_{h}, \mathbf{v}_{h}\right)=\int_{\Gamma} \hat{\eta} \hat{\mathbf{u}}_{h} \cdot \hat{\mathbf{v}}_{h}+\int_{\partial_{D} \Gamma} \gamma h^{-1}\left(\hat{\mathbf{u}}_{h} \cdot \boldsymbol{\tau}_{\Gamma}\right)\left(\hat{\mathbf{v}}_{h} \cdot \boldsymbol{\tau}_{\Gamma}\right), \\
& \hat{b}\left(\hat{p}_{h}, \hat{\mathbf{v}}_{h}\right)=-\int_{\Gamma} \hat{p}_{h}\left(\nabla \cdot \hat{\mathbf{v}}_{h}\right)+\int_{\partial_{D} \Gamma} \hat{p}_{h}\left(\hat{\mathbf{v}}_{h} \cdot \boldsymbol{\tau}_{\Gamma}\right), \\
& \hat{\mathcal{F}}\left(\hat{\mathbf{v}}_{h}, \hat{q}_{h}\right)=\int_{\Gamma} \hat{\boldsymbol{f}}_{v} \cdot \hat{\mathbf{v}}_{h}+\int_{\Gamma}\left(l_{\Gamma} \tilde{f}_{q}+\llbracket \mathbf{u}_{h} \cdot \mathbf{n}_{\Gamma} \rrbracket\right) \hat{q}_{h}-\int_{\partial \Gamma_{N}} \hat{p}_{0}\left(\hat{\mathbf{v}}_{h} \cdot \mathbf{n}\right) .
\end{aligned}
$$

Our finite element method reads as follows: given the normal velocity jump $\llbracket \mathbf{u}_{h} \cdot \mathbf{n}_{\Gamma} \rrbracket$, find $\left(\hat{\mathbf{u}}_{h}, \hat{p}_{h}\right) \in \hat{\mathbf{W}}_{h}$ such that

$$
\begin{gathered}
\hat{\mathcal{C}}\left(\left(\hat{\mathbf{u}}_{h}, \hat{p}_{h}\right),\left(\hat{\mathbf{v}}_{h}, \hat{q}_{h}\right)\right)=\hat{\mathcal{F}}\left(\hat{\mathbf{v}}_{h}, \hat{q}_{h}\right) \quad \forall\left(\hat{\mathbf{v}}_{h}, \hat{q}_{h}\right) \in \hat{\mathbf{W}}_{h}, \\
\quad \text { where } \\
\hat{\mathcal{C}}\left(\left(\hat{\mathbf{u}}_{h}, \hat{p}_{h}\right),\left(\hat{\mathbf{v}}_{h}, \hat{q}_{h}\right)\right)=\hat{a}\left(\hat{\mathbf{u}}_{h}, \hat{\mathbf{v}}_{h}\right)+\hat{b}\left(\hat{p}_{h}, \hat{\mathbf{v}}_{h}\right)-\hat{b}\left(\hat{q}_{h}, \hat{\mathbf{u}}_{h}\right) .
\end{gathered}
$$

Note that problems (3.4) and (5.4) are coupled, since $\mathcal{F}$ depends on the pressure within the fracture, and $\hat{\mathcal{F}}$ on the normal jump of the bulk velocity. We also observe that the bilinear forms $\mathcal{C}$ and $\hat{\mathcal{C}}$ associated to problems (3.4) and (5.4) have exactly the same structure, although they are related to Darcy's problems on domains having different dimensions, and no extended element is introduced in the fracture flow problem. In particular, all boundedness, stability and convergence properties of (3.4) are immediately extended to (5.4) (with $\hat{M}_{\Gamma}=1$, since there is no embedded interface).

\subsection{Iterative solution of the coupled problem}

The reduction of the solution of the coupled problem to successive solutions on decoupled bulk flow and fracture flow problems has the clear advantage of allowing the use of different solvers and possible parallel implementation. As already observed (without convergence analysis) in [15] in the case of matching meshes, this implies exchanging data on the "immersed" interface $\Gamma$ and building suitable Steklov-Poincaré operators acting on interface variables. 
In our case, we follow a similar approach providing some more information about the convergence of the related iterative algorithm. In particular, we introduce the following operators.

- Let $\left(\mathcal{H}_{v}, \mathcal{H}_{q}\right): L^{2}(\Gamma) \rightarrow \mathbf{W}_{h}$ be the (porous bulk) resolvent operator associated to (3.4) corresponding to $u_{0}=0, p_{0}=0, \boldsymbol{f}_{v}=\mathbf{0}, f_{q}=0$ (the only nonhomogeneous datum being the fracture pressure $\hat{z}=\hat{p}_{h}$ ), i.e.

$$
\mathcal{C}\left(\left(\mathcal{H}_{v} \hat{z}, \mathcal{H}_{q} \hat{z}\right),\left(\mathbf{v}_{h}, q_{h}\right)\right)=-\int_{\Gamma} \hat{z} \llbracket \mathbf{v}_{h} \cdot \mathbf{n}_{\Gamma} \rrbracket \quad \forall\left(\mathbf{v}_{h}, q_{h}\right) \in \mathbf{W}_{h} .
$$

- Analogously, let $\left(\hat{\mathcal{H}}_{v}, \hat{\mathcal{H}}_{q}\right): L^{2}(\Gamma) \rightarrow \hat{\mathbf{W}}_{h}$ be the (fracture) resolvent operator corresponding to $\hat{\boldsymbol{f}}_{v}=\mathbf{0}$, $\hat{f}_{q}=0$ (the only nonhomogeneous datum being the trans-fracture normal velocity jump $z=\llbracket \mathbf{u}_{h} \cdot \mathbf{n}_{\Gamma} \rrbracket$ ), i.e.

$$
\hat{\mathcal{C}}\left(\left(\hat{\mathcal{H}}_{v} z, \hat{\mathcal{H}}_{q} z\right),\left(\hat{\mathbf{v}}_{h}, \hat{q}_{h}\right)\right)=\int_{\Gamma} z \hat{q}_{h} \quad \forall\left(\hat{\mathbf{v}}_{h}, \hat{q}_{h}\right) \in \hat{\mathbf{W}}_{h} .
$$

- Denote by $\left(\mathbf{u}_{h}^{0}, p_{h}^{0}\right)$ and $\left(\hat{\mathbf{u}}_{h}^{0}, \hat{p}_{h}^{0}\right)$ respectively the solution of problem (3.4) for $\hat{p}_{h}=0$ and the solution of problem (5.4) for $\llbracket \mathbf{u}_{h} \cdot \mathbf{n}_{\Gamma} \rrbracket=0$.

- Thanks to linearity, we can express the solution of problems (3.4) and (5.4) by

$$
\left(\mathbf{u}_{h}, p_{h}\right)=\left(\mathbf{u}_{h}^{0}, p_{h}^{0}\right)+\left(\mathcal{H}_{v} \hat{p}_{h}, \mathcal{H}_{q} \hat{p}_{h}\right), \quad\left(\hat{\mathbf{u}}_{h}, \hat{p}_{h}\right)=\left(\hat{\mathbf{u}}_{h}^{0}, \hat{p}_{h}^{0}\right)+\left(\hat{\mathcal{H}}_{v} \llbracket \mathbf{u}_{h} \cdot \mathbf{n}_{\Gamma} \rrbracket, \hat{\mathcal{H}}_{q} \llbracket \mathbf{u}_{h} \cdot \mathbf{n}_{\Gamma} \rrbracket\right) .
$$

Lemma 5.1. For all $z, \hat{z} \in L^{2}(\Gamma)$ we have

$$
\left\|\left(\mathcal{H}_{v} \hat{z}, \mathcal{H}_{q} \hat{z}\right)\right\|_{\mathbf{W}_{h}}^{2} \lesssim C\|\hat{z}\|_{L^{2}(\Gamma)}^{2}, \quad\left\|\left(\hat{\mathcal{H}}_{v} z, \hat{\mathcal{H}}_{q} z\right)\right\|_{\hat{\mathbf{W}}_{h}}^{2} \lesssim \hat{C}\|z\|_{L^{2}(\Gamma)}^{2},
$$

with $C=\frac{M_{\Gamma}^{2}}{\xi_{0} \eta_{\Gamma}}, \hat{C}=\hat{\eta}$, where $M_{\Gamma}$ is the constant of Lemma 4.8 .

Proof. By Lemma 4.2 and Theorem 4.10, we get immediately

$$
\left\|\left(\mathcal{H}_{v} \hat{z}, \mathcal{H}_{q} \hat{z}\right)\right\|_{\mathbf{w}_{h}} \lesssim \xi_{0}^{-\frac{1}{2}} M_{\Gamma}\left\|\eta_{\Gamma}^{-\frac{1}{2}} \hat{z}\right\|_{L^{2}(\Gamma)}
$$

and the first estimate in (5.7) follows since $\eta_{\Gamma}$ is constant. The second estimate is obtained analogously.

Now, we reformulate our coupled problem (3.4), (5.4), as an equation for $\hat{p}_{h} \in \hat{Q}_{h}$,

$$
\hat{p}_{h}=\hat{p}_{h}^{0}+\hat{\mathcal{H}}_{q} \llbracket \mathbf{u}_{h} \cdot \mathbf{n}_{\Gamma} \rrbracket=\hat{p}_{h}^{0}+\hat{\mathcal{H}}_{q} \llbracket \mathbf{u}_{h}^{0} \cdot \mathbf{n}_{\Gamma} \rrbracket+\hat{\mathcal{H}}_{q} \llbracket\left(\mathcal{H}_{v} \hat{p}_{h}\right) \cdot \mathbf{n}_{\Gamma} \rrbracket .
$$

Let us introduce the operators $S: L^{2}(\Gamma) \rightarrow L^{2}(\Gamma), \hat{S}: L^{2}(\Gamma) \rightarrow \hat{Q}_{h}$ defined by $S \hat{z}=-\llbracket\left(\mathcal{H}_{v} \hat{z}\right) \cdot \mathbf{n}_{\Gamma} \rrbracket, \hat{S} \hat{z}=\hat{\mathcal{H}}_{q} \hat{z}$, and respectively associated to the bilinear forms

$$
s(\hat{p}, \hat{q}):=-\int_{\Gamma} \llbracket\left(\mathcal{H}_{v} \hat{p}\right) \cdot \mathbf{n}_{\Gamma} \rrbracket \hat{q}, \quad \hat{s}(\hat{p}, \hat{q}):=\int_{\Gamma}\left(\hat{\mathcal{H}}_{q} \hat{p}\right) \hat{q} .
$$

Then, equation (5.8) becomes

$$
\hat{p}_{h}=\hat{S}\left(-S \hat{p}_{h}+\llbracket \mathbf{u}_{h}^{0} \cdot \mathbf{n}_{\Gamma} \rrbracket\right)+\hat{p}_{h}^{0} .
$$

Let $\omega \geq 0$ be a relaxation parameter; we shall first consider the following iterative method for the solution of $(5.10)$,

$$
\hat{p}_{h}^{(k+1)}+\omega\left(\hat{p}_{h}^{(k+1)}-\hat{p}_{h}^{(k)}\right)=\left[\hat{S}\left(-S \hat{p}_{h}^{(k)}+\llbracket \mathbf{u}_{h}^{0} \cdot \mathbf{n}_{\Gamma} \rrbracket\right)+\hat{p}_{h}^{0}\right] .
$$

Theorem 5.2. The following properties hold true.

(i) $S$ is symmetric positive semidefinite and $\hat{S}$ symmetric positive definite on $\hat{Q}_{h}$, i.e. $s\left(\hat{q}_{h}, \hat{q}_{h}\right) \geq 0, \hat{s}\left(\hat{q}_{h}, \hat{q}_{h}\right)>$ $0 \forall 0 \neq \hat{q}_{h} \in \hat{Q}_{h}$. 
(ii) The iterative method (5.11) converges for $\omega \gtrsim M_{\Gamma}^{2} \frac{\hat{\eta}}{\xi_{0} \eta_{\Gamma}}$.

(iii) The convergence rates only depend on $\omega$, and not on $h$, at least asymptotically for $h$ small $\left(M_{\Gamma} \simeq 1\right)$. Moreover, there is an optimal relaxation parameter $\omega_{*}>0$ that maximizes the convergence speed.

Proof. Let us consider each point separately.

(i) By (5.9) and (5.5) with $\hat{z}=\hat{q}_{h}$, we have $b\left(q_{h}, \mathcal{H}_{v} \hat{q}_{h}\right)=0 \forall q_{h} \in Q_{h}, s\left(\hat{p}_{h}, \hat{q}_{h}\right)=a\left(\mathcal{H}_{v} \hat{q}_{h}, \mathcal{H}_{v} \hat{p}_{h}\right)+$ $b\left(\hat{\mathcal{H}}_{q} \hat{q}_{h}, \mathcal{H}_{v} \hat{p}_{h}\right)$, so that

$$
s\left(\hat{p}_{h}, \hat{q}_{h}\right)=a\left(\mathcal{H}_{v} \hat{q}_{h}, \mathcal{H}_{v} \hat{p}_{h}\right) .
$$

This shows that if $a$ is positive definite then $S$ is positive semidefinite. Note also that in our case $a$ is symmetric, and so is $S$. Similarly, by (5.9) and (5.6) we have $\hat{a}\left(\hat{\mathcal{H}}_{v} \hat{p}_{h}, \hat{\mathbf{v}}_{h}\right)+\hat{b}\left(\hat{\mathcal{H}}_{q} \hat{p}_{h}, \hat{\mathbf{v}}_{h}\right)=0 \forall \hat{\mathbf{v}}_{h} \in \hat{\mathbf{V}}_{h}, \hat{s}\left(\hat{p}_{h}, \hat{q}_{h}\right)=$ $-\hat{b}\left(\hat{\mathcal{H}}_{q} \hat{p}_{h}, \hat{\mathcal{H}}_{v} \hat{q}_{h}\right)$, so that taking $\hat{\mathbf{v}}_{h}=\hat{\mathcal{H}}_{v} \hat{q}_{h}$

$$
\hat{s}\left(\hat{p}_{h}, \hat{q}_{h}\right)=\hat{a}\left(\hat{\mathcal{H}}_{v} \hat{q}_{h}, \hat{\mathcal{H}}_{v} \hat{p}_{h}\right) .
$$

We observe that, from (5.6) we have $\int_{\Gamma} \hat{q}_{h}^{2}=\hat{b}\left(\hat{q}_{h}, \hat{\mathcal{H}}_{v} \hat{q}_{h}\right) \forall \hat{q}_{h} \in \hat{Q}_{h}$, which implies $\left\|\hat{q}_{h}\right\|_{L^{2}(\Gamma)} \lesssim \hat{\eta}^{-1}\left\|\hat{\mathcal{H}}_{v} \hat{q}_{h}\right\|_{\hat{\mathbf{V}}_{h}}$. This shows that $\hat{\mathcal{H}}_{v}$ is injective, and so, by equation (5.13), $\hat{S}$ is positive definite. Note also that we cannot upper bound $\left\|\hat{\mathcal{H}}_{v} z\right\|_{\hat{\mathbf{V}}_{h}}^{2}$ by $\hat{a}\left(\hat{\mathcal{H}}_{v} z, \hat{\mathcal{H}}_{v} z\right)$ unless using inverse inequalities, which would give a coercivity constant depending on $h$. Nevertheless, as we will see, we do not need uniform coercivity for $\hat{S}$.

(ii) Introduce the operator $T:=\hat{S} S . T$ is obviously non-negative, thanks to i). Note that $\hat{S}$ and $S$ are bounded; in fact, by (5.7) and (5.12), (5.13), we have $s\left(\hat{p}_{h}, \hat{q}_{h}\right) \leq \sqrt{C}\left\|\hat{p}_{h}\right\|_{L^{2}(\Gamma)}\left\|\hat{q}_{h}\right\|_{L^{2}(\Gamma)}, \hat{s}\left(\hat{p}_{h}, \hat{q}_{h}\right) \leq$ $\sqrt{\hat{C}}\left\|\hat{p}_{h}\right\|_{L^{2}(\Gamma)}\left\|\hat{q}_{h}\right\|_{L^{2}(\Gamma)}$. As a consequence, $T$ is bounded, i.e. $\left(\hat{q}_{h}, T \hat{p}_{h}\right)_{L^{2}(\Gamma)} \leq C_{T}\left\|\hat{p}_{h}\right\|_{L^{2}(\Gamma)}\left\|\hat{q}_{h}\right\|_{L^{2}(\Gamma)}, C_{T}=$ $\sqrt{\hat{C} C}$.

Now, let us focus on the iterative method (5.11). Consider first the case of homogeneous data $\left(p_{h}^{0}=\mathbf{u}_{h}^{0} \cdot \mathbf{n}_{\Gamma}=\right.$ $0)$, i.e. given $\hat{p}_{h}^{(0)}$ compute the sequence defined by

$$
\hat{p}_{h}^{(k+1)}+\omega\left(\hat{p}_{h}^{(k+1)}-\hat{p}_{h}^{(k)}\right)=-T \hat{p}_{h}^{(k)} .
$$

We claim that sequence to be convergent to zero for all $\hat{p}_{h}^{(0)}$. Observing that

$$
\begin{aligned}
\left(\hat{p}_{h}^{(k+1)}, T \hat{p}_{h}^{(k)}\right)_{L^{2}(\Gamma)} & =\left(\hat{p}_{h}^{(k+1)}, T \hat{p}_{h}^{(k+1)}\right)_{L^{2}(\Gamma)}-\left(\hat{p}_{h}^{(k+1)}, T\left(\hat{p}_{h}^{(k+1)}-\hat{p}_{h}^{(k)}\right)\right)_{L^{2}(\Gamma)}, \\
\left(\hat{p}_{h}^{(k+1)}, \hat{p}_{h}^{(k+1)}-\hat{p}_{h}^{(k)}\right)_{L^{2}(\Gamma)} & =\frac{1}{2}\left[\left\|\hat{p}_{h}^{(k+1)}-\hat{p}_{h}^{(k)}\right\|_{L^{2}(\Gamma)}^{2}+\left\|\hat{p}_{h}^{(k+1)}\right\|_{L^{2}(\Gamma)}^{2}-\left\|\hat{p}_{h}^{(k)}\right\|_{L^{2}(\Gamma)}^{2}\right],
\end{aligned}
$$

by multiplying equation (5.14) times $\hat{p}_{h}^{(k+1)}$ and integrating on $\Gamma$, owing to the positivity of $T$, and to the Young inequality, for any $\epsilon>0$ we get

$$
\begin{aligned}
\left\|\hat{p}_{h}^{(k+1)}\right\|_{L^{2}(\Gamma)}^{2}+\frac{\omega}{2}\left[\left\|\hat{p}_{h}^{(k+1)}-\hat{p}_{h}^{(k)}\right\|_{L^{2}(\Gamma)}^{2}+\left\|\hat{p}_{h}^{(k+1)}\right\|_{L^{2}(\Gamma)}^{2}-\left\|\hat{p}_{h}^{(k)}\right\|_{L^{2}(\Gamma)}^{2}\right] & \\
& \leq \epsilon C_{T}\left\|\hat{p}_{h}^{(k+1)}\right\|_{L^{2}(\Gamma)}^{2}+\frac{1}{\epsilon} C_{T}\left\|\hat{p}_{h}^{(k+1)}-\hat{p}_{h}^{(k)}\right\|_{L^{2}(\Gamma)}^{2},
\end{aligned}
$$

where $C_{T}$ is the boundedness constant of $T$ with respect to the $L^{2}(\Gamma)$ norm; note that $C_{T}^{2} \lesssim M_{\Gamma}^{2} \frac{\hat{\eta}}{\xi_{0} \eta_{\Gamma}}$. Taking $\epsilon=1 /\left(2 C_{T}\right)$, we get

$$
\left\|\hat{p}_{h}^{(k+1)}\right\|_{L^{2}(\Gamma)}^{2}+\left(\omega-4 C_{T}^{2}\right)\left\|\hat{p}_{h}^{(k+1)}-\hat{p}_{h}^{(k)}\right\|_{L^{2}(\Gamma)}^{2}+\omega\left\|\hat{p}_{h}^{(k+1)}\right\|_{L^{2}(\Gamma)}^{2} \leq \omega\left\|\hat{p}_{h}^{(k)}\right\|_{L^{2}(\Gamma)}^{2} .
$$

Summing over $k$, exploiting the telescopic terms $\omega\left\|\hat{p}_{h}^{(k+1)}\right\|_{L^{2}(\Gamma)}^{2}$ and $\omega\left\|\hat{p}_{h}^{(k)}\right\|_{L^{2}(\Gamma)}^{2}$, we conclude that for $\omega>4 C_{T}^{2}$

$$
\sum_{k=0}^{\infty}\left\|\hat{p}_{h}^{(k)}\right\|_{L^{2}(\Gamma)}^{2} \leq \omega\left\|\hat{p}_{h}^{(0)}\right\|_{L^{2}(\Gamma)}^{2} .
$$


As a consequence, $\left\|\hat{p}_{h}^{(k)}\right\|_{L^{2}(\Gamma)} \rightarrow 0$ as $k \rightarrow \infty$ for any $\hat{p}_{h}^{(0)} \in \hat{Q}_{h}$. This in turns implies that the spectral radius of the iteration operator associated with equation (5.10) is strictly smaller than one, and the iterative method is convergent also for non homogeneous data.

(iii) We observe that since $\hat{S}$ is positive definite, it is invertible; the iterative method (5.11) can be recast in the following preconditioned Richardson form,

$$
\hat{p}_{h}^{(k+1)}=\hat{p}_{h}^{(k)}+\alpha \hat{R}^{-1}\left[\hat{b}_{h}-R \hat{p}_{h}^{(k)}\right], \quad \hat{b}_{h}=\llbracket \mathbf{u}_{h}^{0} \cdot \mathbf{n}_{\Gamma} \rrbracket+\hat{S} \hat{p}_{h}^{0}, \quad \alpha=\frac{1}{1+\omega},
$$

where the linear operator $R:=\hat{R}+S$ is preconditioned by $\hat{R}=\hat{S}^{-1}$. Note that $R$ and $\hat{R}$ are both symmetric positive definite, and $\hat{R}^{-1} R=I+T$. This provides us with a further convergence proof. In fact we have

$$
(\hat{z}, \hat{z})_{L^{2}(\Gamma)} \leq(\hat{z}, \hat{z})_{L^{2}(\Gamma)}+(\hat{z}, T \hat{z})_{L^{2}(\Gamma)}=\left(\hat{z}, \hat{R}^{-1} R \hat{z}\right)_{L^{2}(\Gamma)} \leq\left(1+C_{T}^{2}\right)(\hat{z}, \hat{z})_{L^{2}(\Gamma)},
$$

and we can bound the Rayleigh quotient of the preconditioned iteration operator as

$$
1 \lesssim \frac{\left(\hat{z}, \hat{R}^{-1} R \hat{z}\right)_{L^{2}(\Gamma)}}{(\hat{z}, \hat{z})_{L^{2}(\Gamma)}} \lesssim 1+M_{\Gamma}^{2} \frac{\hat{\eta}}{\xi_{0} \eta_{\Gamma}}
$$

From the convergence theory of preconditioned Richardson iterations (see for instance [18], Chap. 2) we see that the iterative method is convergent for $0<\alpha \lesssim\left(1+M_{\Gamma}^{2} \frac{\hat{\eta}}{\xi_{0} \eta_{\Gamma}}\right)^{-1}$, confirming our previous estimate for $\omega$; moreover, we have that an optimal convergence parameter $\alpha_{*}$ exists, and, more importantly, the convergence rates of the iterative methods are asymptotically independent of $h$, since the spectral bounds above are so. A confirmation of this fact will be given also by numerical experiments (see Tab. 2 in Sect. 6). Approximating the minimum and maximum eigenvalues of $\hat{R}^{-1} R$ respectively by 1 and $1+m, m=M_{\Gamma}^{2} \frac{\hat{\eta}}{\xi_{0} \eta_{\Gamma}}$, we have $\alpha_{*} \simeq \frac{2}{2+m}$ (or $\omega_{*} \simeq m / 2$ ), and, at each iteration, the error is reduced by a factor that in the optimal case is about $\frac{m}{2+m}$; we thus expect the convergence rates to degrade when $m$ is big.

As a consequence of Theorem 5.2, the coupled problem is well-posed. Moreover, the theorem provides an iterative algorithm to solve the coupled problem, which is (asymptotically) optimal with respect to $h$. From the computational point of view, it is also convenient to write the iterative method (5.11) as follows.

For $k=0,1, \ldots$ :

1. Given $\hat{p}_{h}^{(k)}$, solve the bulk flow and compute $\mathbf{u}_{h}^{(k)}=\mathbf{u}_{h}^{0}+\mathcal{H}_{v} \hat{p}_{h}^{(k)}$;

2. Solve the fracture flow and compute $\hat{p}_{h}^{\left(k+\frac{1}{2}\right)}=\hat{p}_{h}^{0}+\hat{\mathcal{H}}_{q} \llbracket \mathbf{u}_{h}^{(k)} \cdot \mathbf{n}_{\Gamma} \rrbracket$;

3. Update the fracture pressure:

$$
\hat{p}_{h}^{(k+1)}=\theta \hat{p}_{h}^{(k)}+(1-\theta) \hat{p}_{h}^{\left(k+\frac{1}{2}\right)}, \quad \theta=\frac{\omega}{1+\omega} .
$$

\section{NumeriCAL EXPERIMENTS AND APPLICATIONS}

\subsection{Convergence of the decoupled problem}

Let $\Omega=[0,1]^{2}, R \in(0,1)$ and $\Gamma=\left\{(x, y) \in \Omega: r^{2}=x^{2}+y^{2}=R^{2}\right\}$. We consider problem (2.1) with $\eta=1$, $\eta_{\Gamma}=\frac{2}{3} R, \Gamma_{N}=\partial \Omega, \Gamma_{D}=\emptyset$, and

$$
f_{q}=\left\{\begin{array}{ll}
2 / R^{2} & \text { if } r^{2}>R^{2}, \\
4 / R^{2} & \text { if } r^{2}<R^{2},
\end{array} \quad p_{0}= \begin{cases}\frac{r^{2}}{2 R^{2}}+\frac{3}{2} & \text { if } r^{2}>R^{2}, \\
\frac{r^{2}}{R^{2}} & \text { if } r^{2}<R^{2} .\end{cases}\right.
$$



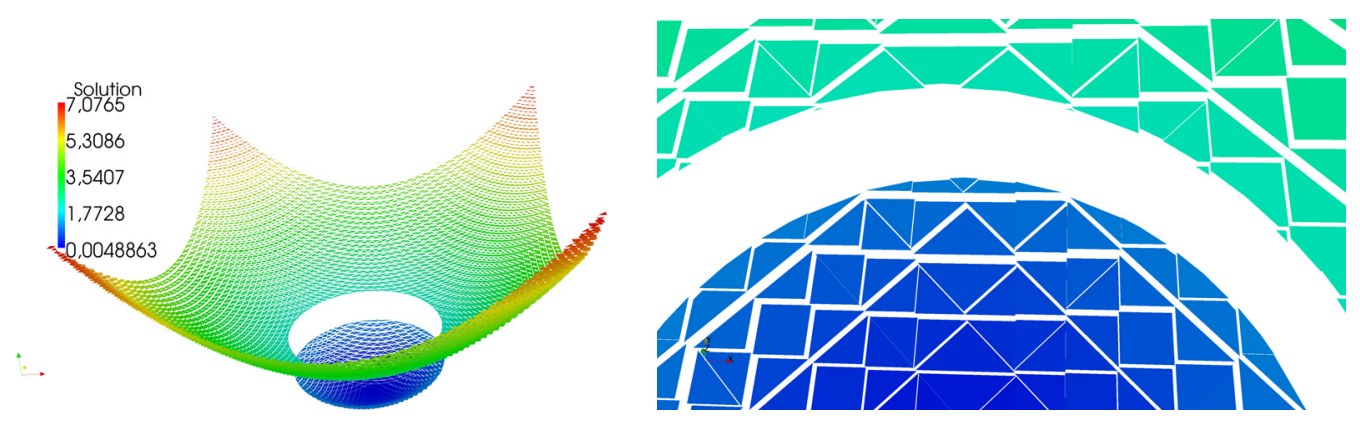

FiguRE 5. Left: numerical solution to the uncoupled Darcy's problem. Right: zoom on the cut region $\mathcal{G}_{h}$ showing the extended pressure elements.

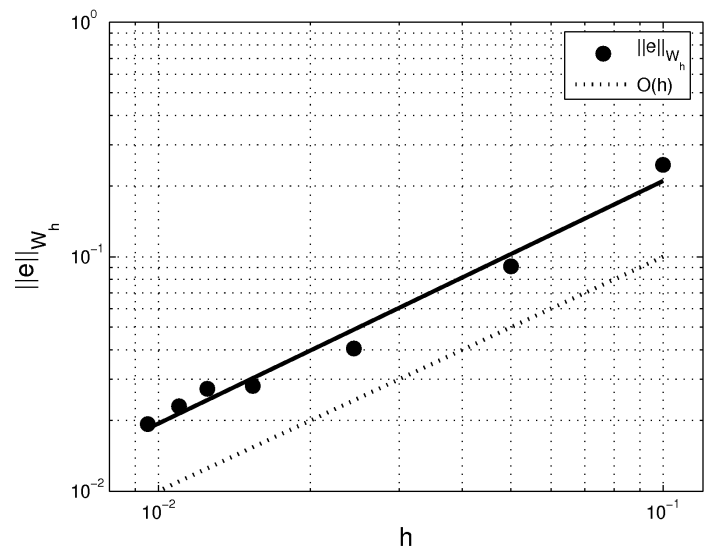

FiguRE 6. Convergence of the error $\left\|\left(\mathbf{u}-\mathbf{u}_{h}, p-p_{h}\right)\right\|_{\mathbf{W}_{h}}$ for the uncoupled problem.

In this case, the boundary data $p_{0}$ provides also the exact pressure solution $p$ and velocity $\mathbf{u}=-\nabla p$. The pressure in the fracture is imposed, $\hat{p}=\frac{19}{12}$, and choosing $\xi=\frac{3}{4}$ yields $\llbracket \mathbf{u} \cdot \mathbf{n}_{\Gamma} \rrbracket=\frac{1}{R}$. The numerical solution, computed on a grid of about 6500 elements is shown in Figure 5. Figure 6 shows the error in the norm defined by (4.2) for different $h$, confirming the estimate (4.13), i.e. the error decreases linearly with the mesh size.

\subsection{Coupled bulk-fracture flow problems}

The full coupled system for the bulk and fracture flow reads

$$
\left[\begin{array}{cccc}
A & B^{T} & 0 & E \\
-B & 0 & 0 & 0 \\
0 & 0 & \hat{A} & \hat{B}^{T} \\
-E^{T} & 0 & -\hat{B} & 0
\end{array}\right]\left[\begin{array}{c}
\mathbf{u} \\
\mathbf{p} \\
\hat{\mathbf{u}} \\
\hat{\mathbf{p}}
\end{array}\right]=\left[\begin{array}{c}
\boldsymbol{f}_{v} \\
f_{q} \\
\hat{f}_{v} \\
\tilde{f}_{q}
\end{array}\right]
$$

where the blocks $E$ and $E^{T}$ are due to the interface conditions that couple the two problems. In particular the entries of $E$ are $e_{i j}=\int_{\Gamma}\left(\hat{q}_{h}\right)_{j} \llbracket\left(\mathbf{v}_{h}\right)_{i} \cdot \mathbf{n}_{\Gamma} \rrbracket$. Since the basis functions defining $e_{i j}$ are related to different meshes (although the integral is computed on $\Gamma$, hence using the fracture mesh), in general an interpolation has to be performed between the bulk mesh $\mathcal{T}_{h}$ covering $\Omega$ and the fracture mesh $\hat{\mathcal{T}}_{h}$ on $\Gamma$. The system can be solved either directly or using the iterative method of Section 5. We observe that also for the coupled problem, by changing sign in the mass conservation equations the system can be recast in a symmetric (indefinite) form. 


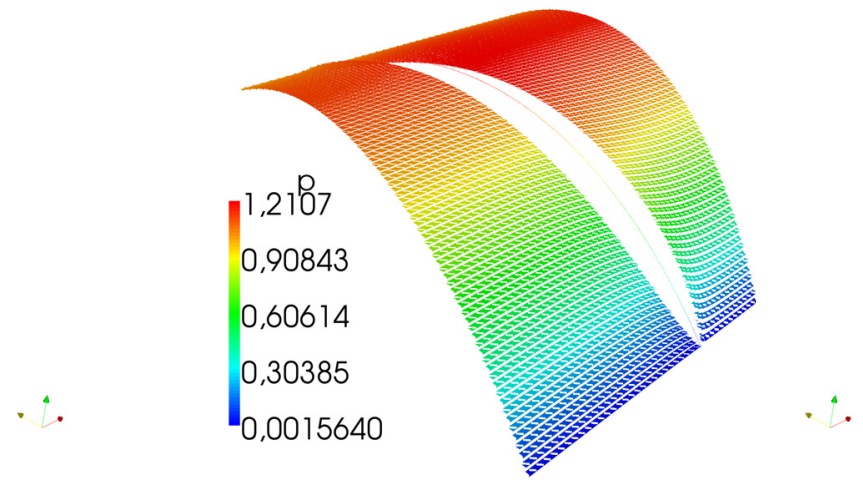

(a)

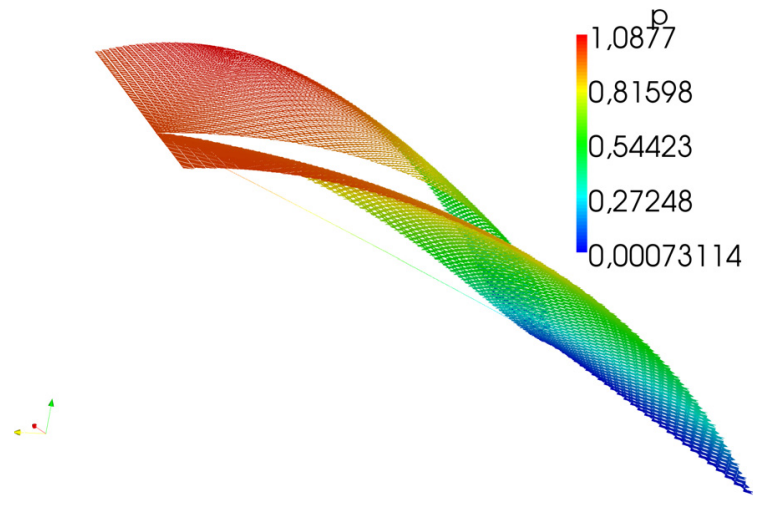

(c)

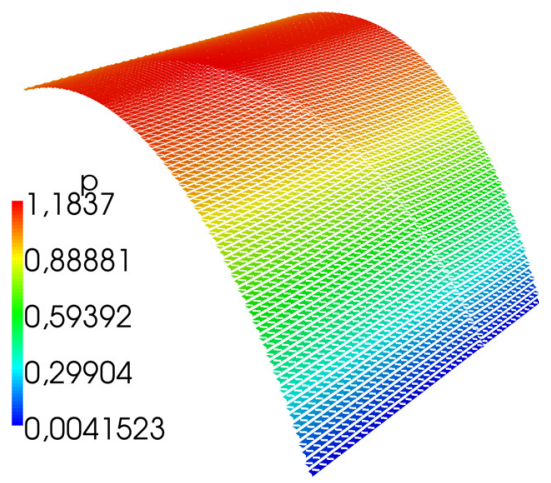

(b)

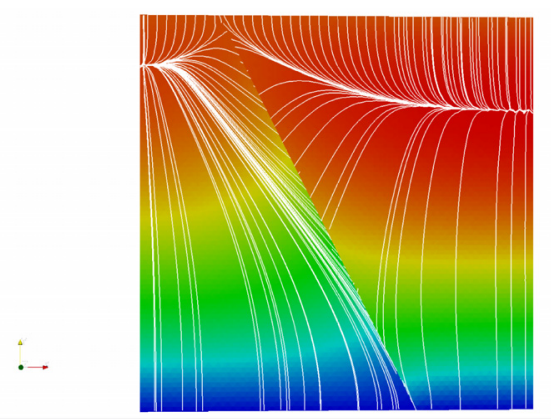

(d)

FiguRe 7. Pressure in the porous domain and in the fracture for different values of parameters, namely (a) $\eta=1, \eta_{\Gamma}=1, \hat{\eta}=100$; (b) $\eta=1, \eta_{\Gamma}=0.01, \hat{\eta}=100 ;$ (c) $\eta=1, \eta_{\Gamma}=1$, $\hat{\eta}=1$. In (d): streamlines corresponding to case (c), where the fracture with a high tangential permeability becomes a preferential path for the flow.

We here consider a two-dimensional problem in a square domain cut by a slanting fracture and analyze the solution for different values of the inverse permeabilities $\eta, \eta_{\Gamma}, \hat{\eta}$. Let $\Omega=[0,1]^{2}, \Gamma=\{(x, y) \in \Omega: y+2 x=1.4\}$, $\Gamma_{D}=\{0,1\} \times[0,1]$, and $\Gamma_{N}=[0,1] \times\{0,1\}$. The bulk flow and the flow in the fracture are described by equations (2.1) and (2.5), with $f_{q}=\hat{f}_{q}=4, p_{0}=y$, and $l_{\Gamma}=0.01$, considering full Neumann boundary conditions $\hat{p}=y$ on $\partial_{N} \Gamma=\partial \Gamma$, coupled by the interface conditions $(2.3)$ with $\xi=0.75$.

Figure 7 represents the numerical solutions for different choices of the parameters. In case (a) $\eta_{\Gamma}=1$, thus the normal permeability $\mathrm{K}_{\Gamma, n}=\frac{l_{\Gamma}}{\eta_{\Gamma}}$ is low and there is a pressure jump across $\Gamma$, while in case (b) pressure is nearly continuous across $\Gamma$ thanks to the high transverse permeability. In case (c) the fracture is very permeable in the tangential direction. As a consequence $\hat{p}$ is almost linear and the streamlines are directed towards $\Gamma$ as shown in (d), because the fracture tends to become a preferential path for the flow. Finally, Figure 8 shows analogous results in a three-dimensional domain cut by a planar fracture for the choices of the parameters (a) and (c).

\subsection{Convergence of the iterative scheme}

The results of the coupled problem obtained with the iterative scheme (5.16) can be compared with the monolithic approach to assess experimentally the convergence of the iterative procedure. Figure 9 shows the 


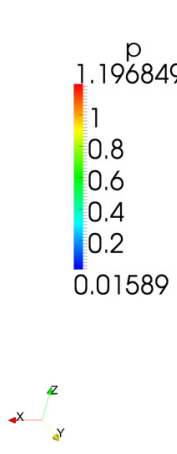

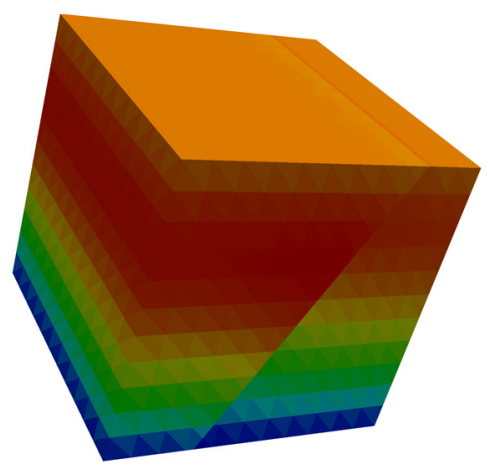

(a)

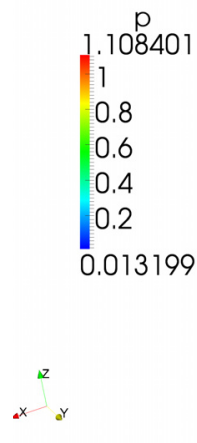

Figure 8. Pressure in a 3D porous domain cut by a plane for different values of parameters, namely (a) $\eta=1, \eta_{\Gamma}=1, \hat{\eta}=100$ and (b) $\eta=1, \eta_{\Gamma}=1, \hat{\eta}=1$. Pressure is imposed on the top and bottom boundaries, while the normal velocity is set to zero on the remaining faces.
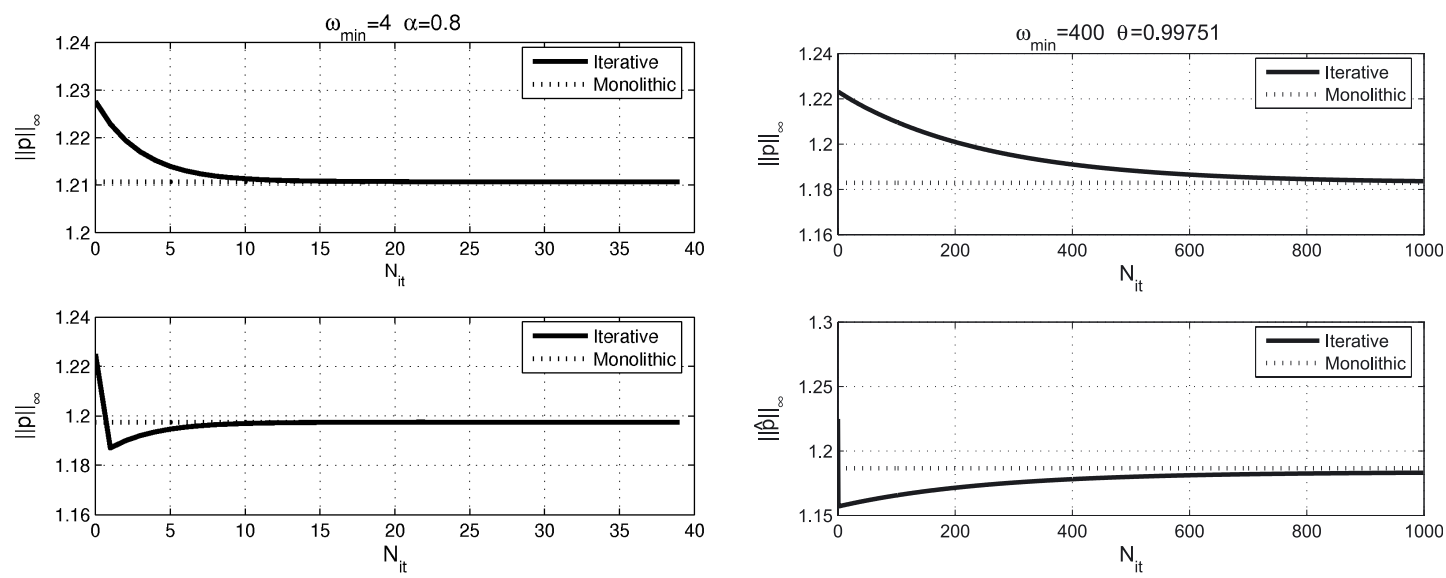

(a)

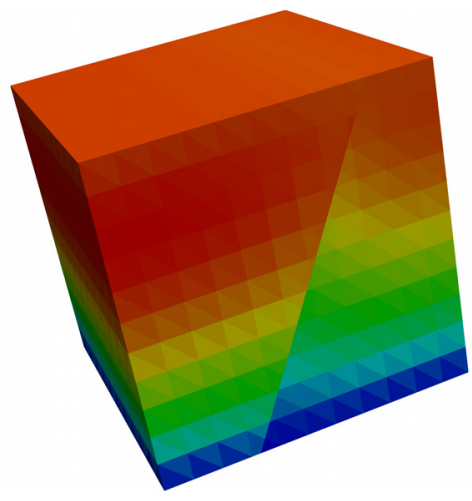

(b)

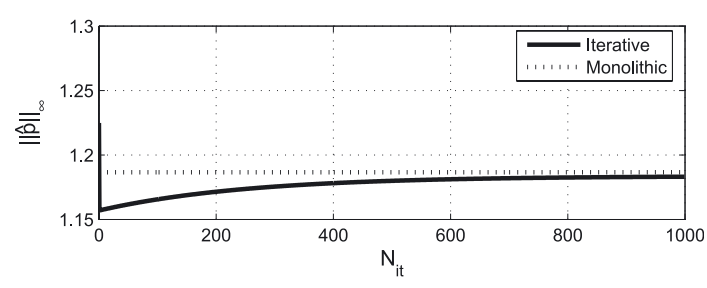

(b)

Figure 9. Convergence of the iterative scheme (compared to the monolithic solution) for different values of the parameters: (a) $\hat{\eta} / \eta_{\Gamma}=1, \theta=0.8$, (b) $\hat{\eta} / \eta_{\Gamma}=100, \theta=0.99751$.

max norm of the pressure ( $p$ and $\hat{p}$ ) as a function of the number of iterations compared to the "exact" one computed with a monolithic approach for the test cases presented in the previous section. The three cases are characterized by different rates of convergence since the relaxation parameter depends on the $\hat{\eta} / \eta_{\Gamma}$ ratio (assuming $M_{\Gamma} \simeq 1$ ). Taking $\xi=0.75$, and thus $\xi_{0}=0.125$ for case a), we choose $\omega$ as in Theorem 5.2 , resulting in $\theta=0.8$. Iterations stabilize, in the maximum norm, at about 20 . Case b) is critical; having $\hat{\eta} / \eta_{\Gamma}=100$ we take $\theta>0.9975$ and indeed after 1000 iterations the solution has not reached convergence yet. Notice that in general we expect that the smaller $\hat{\eta} / \eta_{\Gamma}$ the faster the convergence of the iterative method (with optimal relaxation parameters, see the proof of Theorem 5.2). For instance, for $\hat{\eta} / \eta_{\Gamma}=0.01$, our theory predicts that a very small relaxation parameter $(\theta \simeq 0.04)$ is sufficient. Correspondingly, the convergence of the iterative procedure is very fast and after only a couple of iterations convergence is achieved. 
TABLE 2. Number of iterations of (5.16) as a function of $\theta$ for different mesh sizes. Note the $h$-independent optimal parameter $\theta=\theta_{*} \simeq 0.4$, corresponding to the optimal acceleration parameter $\alpha_{*}$ of the Richardson iterations (5.15).

\begin{tabular}{c|c|c|c}
\hline$\theta^{N_{h}}$ & 882 & 3362 & 13122 \\
\hline 0.1 & 13 & 14 & 13 \\
0.2 & 9 & 9 & 9 \\
0.3 & 6 & 6 & 6 \\
0.4 & 4 & 4 & 4 \\
0.5 & 6 & 6 & 6 \\
0.6 & 9 & 9 & 9 \\
0.7 & 13 & 13 & 13 \\
0.8 & 21 & 22 & 21 \\
0.9 & 44 & 47 & 45 \\
\hline
\end{tabular}

Although only values of $\omega$ chosen according to Theorem 5.2 are guaranteed to ensure convergence, the iterative scheme may converge for smaller $\omega$, i.e. for smaller relaxation parameters $\theta$. Let us consider case (b) and impose values of $\theta$ ranging from 0 to 1 irrespective of the constraint on $\omega$. The iterations to converge to a normalized error in the max norm below $10^{-5}$ are reported in Table 2 for three different meshes, with $h$ respectively about $0.05,0.025,0.0125$. The results show that $\theta_{*}=0.4$ is the optimal value for $\theta$ for the three grids, and that both the number of iterations and $\theta_{*}$ are minimally affected by $h$.

\section{Conclusions}

In this work we proposed an unfitted, mixed finite element method for the numerical approximation of Darcy's flows in fractured porous media. In particular, fractures were treated as interfaces using suitable reduced models [1] and coupling conditions. Owing to an enrichment of the finite element basis on elements cut by the interface, the proposed formulation is able to handle independent meshes for the bulk flow and the fracture flow. We analyzed the abstract properties of the method from stability to convergence. We studied the algebraic properties associated to the resolution of the linear system, and introduced two preconditioners. The first one turned out to be optimal with respect both the mesh size and interface location. We also introduced a much simpler (diagonal) preconditioner, which is optimal with respect to the interface location only. This fact indicates that matrix equilibration is an effective tool to keep the condition number of the discrete problem bounded irrespective of the interface position. Finally, we proposed an iterative method to solve the coupled bulk/fracture flows, analyzed its convergence properties, and reported examples of computations in 2 and 3 dimensions.

The capability of handling independent meshes is a key advantage of our approach, which can express its full potential in applications with variable interfaces, for instance for scenario analysis or in time dependent problems with a mobile interface. Several interesting points are still to be investigated: for instance, the case of branching fractures, or a different numerical technique to impose the coupling conditions (e.g. using suitable Lagrange multipliers, or the Nitsche's method).

\section{REFERENCES}

[1] C. Alboin, J. Jaffré, J.E. Roberts and C. Serres, Modeling fractures as interfaces for flow and transport in porous media, in Fluid flow and transport in porous media: mathematical and numerical treatment (South Hadley, MA, 2001), Contemp. Math., Amer. Math. Soc. 295 (2002) 13-24.

[2] P. Angot, F. Boyer and F. Hubert, Asymptotic and numerical modelling of flows in fractured porous media. ESAIM: M2AN 43 (2009) 239-275.

[3] T. Arbogast, L.C. Cowsar, M.F. Wheeler and I. Yotov, Mixed finite element methods on nonmatching multiblock grids. SIAM J. Numer. Anal. 37 (2000) 1295-1315 (electronic).

[4] D.N. Arnold, R.S. Falk and R. Winther, Preconditioning in H(div) and applications. Math. Comp. 66 (1997) $957-984$. 
[5] R. Becker, P. Hansbo and R. Stenberg, A finite element method for domain decomposition with non-matching grids. ESAIM: M2AN 37 (2003) 209-225.

[6] R. Becker, E. Burman and P. Hansbo, A Nitsche extended finite element method for incompressible elasticity with discontinuous modulus of elasticity. Comput. Methods Appl. Mech. Eng. 198 (2009) 3352-3360.

[7] I.I. Bogdanov, V.V. Mourzenko, J.-F. Thovert and P.M. Adler, Two-phase flow through fractured porous media. Phys. Rev. E 68 (2003) 026703.

[8] F. Brezzi and M. Fortin, Mixed and hybrid finite element methods, Springer Series in Computational Mathematics, SpringerVerlag, New York 15 (1991).

[9] E. Burman and P. Hansbo, A unified stabilized method for Stokes' and Darcy's equations. J. Comput. Appl. Math. 198 (2007) $35-51$.

[10] C. D'Angelo and P. Zunino, A finite element method based on weighted interior penalties for heterogeneous incompressible flows. SIAM J. Numer. Anal. 47 (2009) 3990-4020.

[11] C. D'Angelo and P. Zunino, Robust numerical approximation of coupled stokes and darcy flows applied to vascular hemodynamics and biochemical transport. ESAIM: M2AN 45 (2011) 447-476.

[12] N. Frih, J.E. Roberts and A. Saada, Modeling fractures as interfaces: a model for Forchheimer fractures. Comput. Geosci. 12 (2008) 91-104.

[13] V. Girault and P.A. Raviart, Finite element methods for Navier-Stokes equations, Springer Series in Computational Mathematics, Springer-Verlag, Berlin 5 (1986).

[14] A. Hansbo and P. Hansbo, An unfitted finite element method, based on Nitsche's method, for elliptic interface problems. Comput. Methods Appl. Mech. Eng. 191 (2002) 5537-5552.

[15] V. Martin, J. Jaffré and J.E. Roberts, Modeling fractures and barriers as interfaces for flow in porous media. SIAM J. Sci. Comput. 26 (2005) 1667-1691 (electronic).

[16] N. Moës, J. Dolbow and T. Belytschko, A finite element method for crack growth without remeshing. Internat. J. Numer. Methods Eng. 46 (1999) 131-150.

[17] C.E. Powell and D. Silvester, Optimal preconditioning for Raviart-Thomas mixed formulation of second-order elliptic problems. SIAM J. Matrix Anal. Appl. 25 (2003) 718-738 (electronic).

[18] A. Quarteroni and A. Valli, Numerical Aproximation of Partial Differential Equations. Springer (1994).

[19] A. Reusken, Analysis of an extended pressure finite element space for two-phase incompressible flows. Comput. Vis. Sci. 11 (2008) 293-305.

[20] P. Zunino, L. Cattaneo and C.M. Colciago, An unfitted interface penalty method for the numerical approximation of contrast problems. Appl. Num. Math. 61 (2011) 1059-1076. 